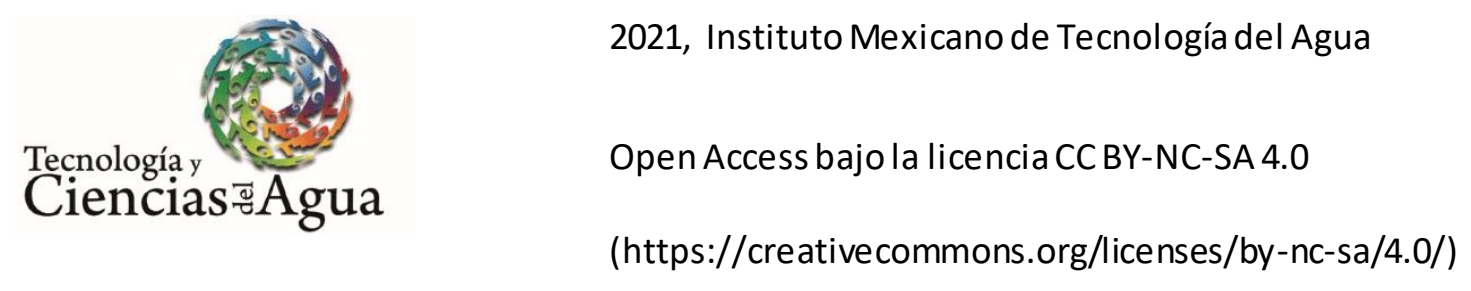

DOI: $10.24850 /$ j-tyca-2021-03-10

Notas

\title{
Calidad de agua del río Puyo y afluentes, Pastaza, Ecuador
}

\section{Water quality of Puyo River and tributaries, Pastaza, Ecuador}

Ricardo Vinicio Abril-Saltos ${ }^{1}$, ORCID: https://orcid.org/0000-0003-15444360

Patricia Abigail Armas-Chugcho²

Washington Patricio Chamorro 3

Víctor Eduardo Toscano-Salazar ${ }^{4}$

Darwin Javier Sucoshañay-Villalva5, ORCID: https://orcid.org/0000-00027724-1190

Antonio Fabricio Ríos-Rodríguez ${ }^{6}$, ORCID: https://orcid.org/0000-00016162-8086

1Universidad Estatal Amazónica, Pastaza, Ecuador, rvabril@uea.edu.ec

2Gobierno Autónomo Descentralizado Provincial de Pastaza, Pastaza, Ecuador, ambiente_armas@hotmail.com

${ }^{3}$ Gobierno Autónomo Descentralizado Provincial de Pastaza, Pastaza, Ecuador, screesh1977@hotmail.com

${ }^{4}$ Gobierno Autónomo Descentralizado Provincial de Pastaza, Pastaza, Ecuador, max_yanempa@hotmail.com 


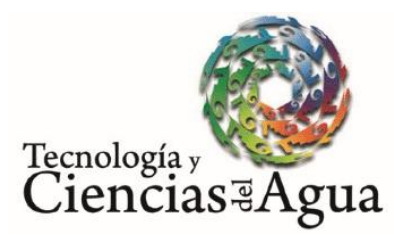

2021, Instituto Mexicano de Tecnología del Agua

Open Access bajo la licencia CCBY-NC-SA 4.0

(https://creativecommons.org/licenses/by-nc-sa/4.0/)

5Universidad Estatal Amazónica, Pastaza, Ecuador, dj.sucoshanayv@uea.edu.ec

6Gobierno Autónomo Descentralizado Provincial de Pastaza, Pastaza, Ecuador, riosfabricio2014@gmail.com

Autor para correspondencia: Ricardo Vinicio Abril-Saltos, rvabril@uea.edu.ec

\section{Resumen}

Esta investigación se desarrolló en la subcuenca del río Puyo, Provincia de Pastaza, Ecuador, en los años 2015 y 2018, y tuvo como objetivo caracterizar la calidad ambiental de agua en diferentes sectores de la subcuenca del río Puyo, incluyendo los principales afluentes. Se clasificaron las zonas en alta, urbana y baja. Se realizó un monitoreo en el cauce principal y sus afluentes en 28 puntos, registrándose valores de conductividad, $\mathrm{pH}$, temperatura, saturación de oxígeno, demanda química de oxígeno, demanda bioquímica de oxígeno, y coliformes totales y fecales; se aplicaron 413 cuestionarios para identificar los principales tipos de actividades que se desarrollan en las zonas aledañas al curso principal y afluentes. Los resultados mostraron diferencias en las concentraciones de los distintos parámetros en las fechas y puntos de monitoreo, reportando mayores concentraciones de $\mathrm{DBO}_{5}$, coliformes totales y fecales en las zonas del área urbana. El análisis de correlación mostró relaciones significativas entre las variables estudiadas, con excepción de la saturación de oxígeno. El análisis de varianza mostró diferencias significativas entre las zonas para la conductividad eléctrica, y concentración de coliformes fecales y totales. Los resultados revelan que la mayoría de aguas residuales se depositan de 


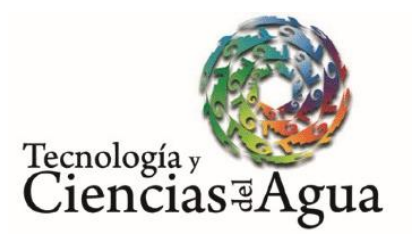

2021, Instituto Mexicano de Tecnología del Agua

Open Access bajo la licencia CC BY-NC-SA 4.0

(https://creativecommons.org/licenses/by-nc-sa/4.0/)

forma directa en el sistema de alcantarillado y en el cauce principal del río. Se concluye que el comportamiento de las diferentes variables registradas difiere entre el cuerpo principal y afluentes, incrementando las concentraciones de coliformes totales y fecales a partir de la zona urbana en el cuerpo principal, y difiriendo el comportamiento del resto de parámetros en las tres zonas.

Palabras clave: contaminación hídrica, agua, población.

\section{Abstract}

This research was developed in the sub-basin of the Puyo River, Province of Pastaza, Ecuador, in the years 2015 and 2018 had as objective: To characterize the environmental quality of water, in different sectors of the sub-basin of the Puyo River, including the main tributaries. The zones were classified as high, urban and low. A monitoring was carried out in the main channel and its tributaries in 28 points, registering values of conductivity, $\mathrm{pH}$, temperature and oxygen saturation, chemical oxygen demand, biochemical oxygen demand, total and fecal coliforms, and 413 surveys were applied to identify the Main types of activities that take place in the areas surrounding the main course and tributaries. The results showed differences in the concentrations of the different parameters in the dates and monitoring points, reporting higher concentrations of BOD5, total and fecal coliforms, the areas of the urban area. The correlation analysis showed significant relationships between the variables studied except for oxygen saturation. The analysis of variance showed significant differences between the zones for electrical conductivity, concentration of fecal and total coliforms. Surveys reveal that the majority of wastewater is deposited directly into the sewer system and the main channel of the river. It is concluded that the behavior of the different registered variables differs 
between the main body and tributaries, increasing the concentrations of total Coliforms, fecal from the urban area in the main body and differing the behavior of the rest of the parameters in the three zones.

Keywords: Water pollution, water, population.

Recibido: 25/03/2019

Aceptado: $25 / 07 / 2020$

\section{Introducción y objetivos}

El manejo del agua históricamente se enfocó en suplir las necesidades humanas para alimentación y riego, conduciendo a un descuido en la disposición de agua fresca para especies y ecosistemas (Ritcher, Mathews, Harrison, \& Wigington, 2003), donde los ríos también son fuente de recreación y esparcimiento. La urbanización presenta efectos en ecosistemas fluviales en zonas ribereñas, generando cambios en la estructura del lecho y aumento de la carga de contaminantes; esto afecta las funciones ecosistémicas del curso hídrico. Otras consecuencias son la impermeabilización de las áreas de drenaje y captaciones en las cabeceras de los ríos (Rörig et al., 2007).

El cantón Pastaza, ubicado en la provincia de Pastaza, Ecuador, tiene como principal fuente hídrica el río Puyo, el cual presenta influencia de 


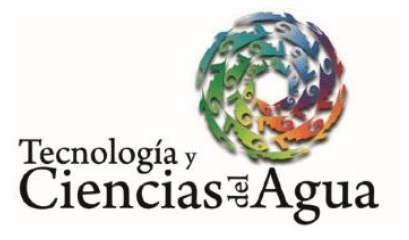

2021, Instituto Mexicano de Tecnología del Agua

Open Access bajo la licencia CCBY-NC-SA 4.0

(https://creativecommons.org/licenses/by-nc-sa/4.0/)

acuerdo con las proyecciones al año 2015 en el cantón Pastaza a 33550 habitantes de la zona urbana (Instituto Nacional de Estadísticas y Censos, 2010); en las zonas rurales, a 5970 habitantes de la parroquia Tarqui (GADPR Tarqui, 2014); 1027 habitantes de la parroquia Fátima (GADPR Fátima, 2015); 232 habitantes de la parroquia Pomona (GADPR Pomona, 2015); 1813 habitantes de la parroquia Veracruz (GADPR Veracruz, 2014) y del cantón Mera; 10310 habitantes de la parroquias Shell (GADPR Shell, 2015), y 1159 habitantes de la parroquia Madre Tierra (GADPR Madre Tierra, 2015); se identifican en toda el área de influencia de la subcuenca del río un aproximado de 60130 habitantes.

El río presenta dos ramales principales, conocidos como Puyo y Pindo, los cuales se unen en el sector Unión Base. En su cuenca media se desarrollan actividades turísticas, deportivas y de conservación de ecosistemas; atraviesa zonas rurales y urbanas, donde las aguas residuales no tienen tratamiento; esto incide en sus características físico-químicas e incrementa la importancia de conocer la calidad ambiental de las aguas de este curso hídrico, y las relaciones existentes entre las variables físicoquímicas y microbiológicas con los caudales presentados.

El presente trabajo se desarrolló en Ecuador, provincia de Pastaza, cantones Mera y Pastaza, en la subcuenca del río Puyo, del cual la población del cantón Pastaza se abastece para sus requerimientos de agua, donde en su curso principal se presentan dos diques ubicados en los ramales Puyo y Pindo, y también en sus afluentes donde se localizan cuatro diques turísticos. Su cuenca presenta un área de $355 \mathrm{~km}^{2}$ y una longitud total de cauce de $41.4 \mathrm{~km}$. Además, en varios sectores de su curso principal y afluentes se encuentran balnearios naturales donde se realizan actividades recreativas. 


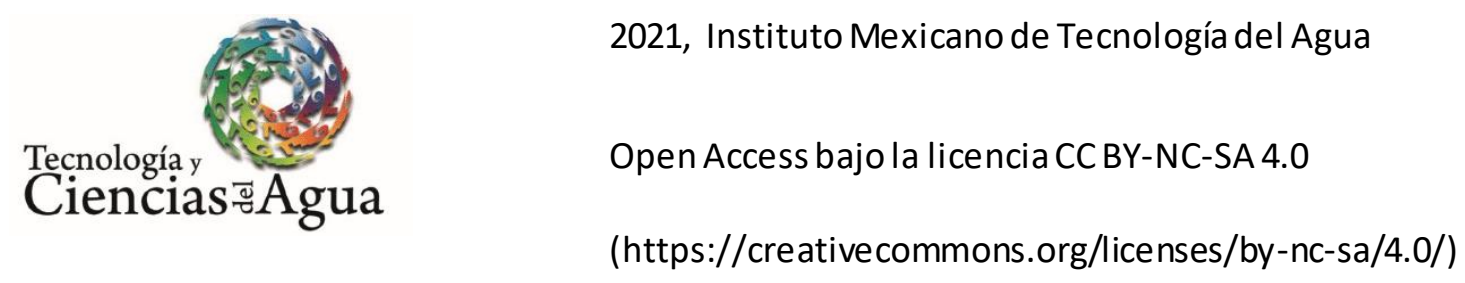

Este trabajo se efectuó entre los años 2015-2018. Se enfocó principalmente en el campo temático de calidad del agua y su objetivo fue caracterizar la calidad ambiental de agua en diferentes sectores de la subcuenca del río Puyo, incluyendo los principales afluentes.

\section{Materiales y métodos}

Se realizó un monitoreo del año 2015 al año 2018 en 28 puntos: 10 ubicados en el curso principal y 18 en sus afluentes (Figura 1), cubriendo una longitud de cauce de $9.2 \mathrm{~km}$ en el ramal Pindo y $17 \mathrm{~km}$ en el ramal Puyo. Se tomaron muestras compuestas (Jadhav \& Purohit, 2008), registrando valores in situ de conductividad, $\mathrm{pH}$ y saturación de oxígeno (AWWA Staff, 2010). Se recolectaron muestras para el análisis de demanda bioquímica de oxígeno (DBO5) (Standard Methods No 5210B); demanda química de oxígeno ( $\mathrm{HACH}$ 8000) (Hach, 2014); coliformes totales (Standard Methods No 9222B) (Rice, Baird, Eaton, \& Clesceri, 2012), y coliformes fecales (Standard Methods No 9222 D y 9222I). En la Tabla 1 se reportan coordenadas geográficas, altura, longitud del cauce y gasto base para épocas de precipitación normal y baja precipitación. 

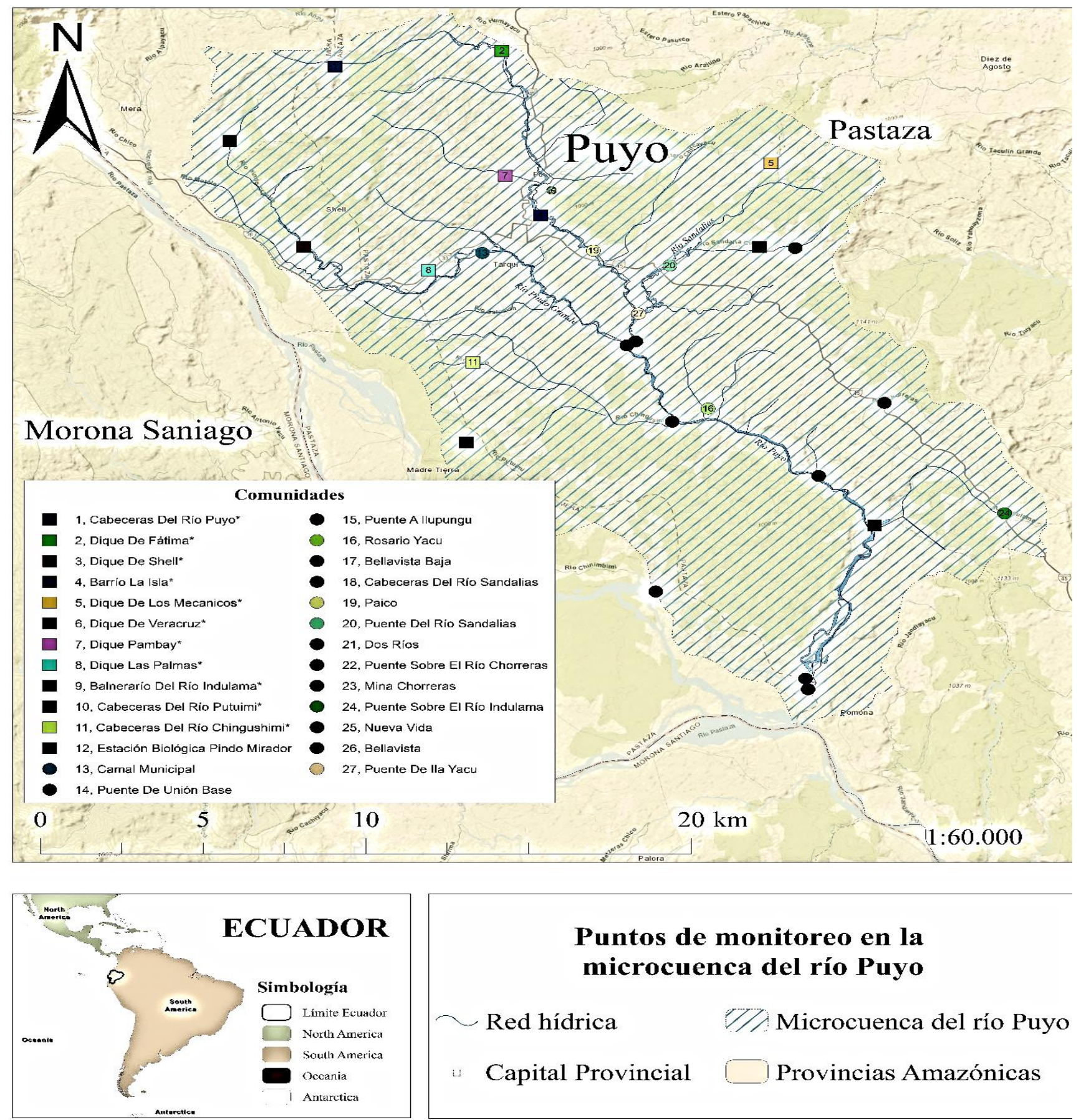

\section{Puntos de monitoreo en la microcuenca del río Puyo}

$\sim$ Red hídrica Microcuenca del río Puyo

Capital Provincial Provincias Amazónicas

DATUM: WGS 84

Proyección: UTM 18S

Fuente: USIG UEA - SNI
Escala
$1: 80.000 .000$

Universidad Estatal $\wedge$ mazónica

\section{usik?}

Figura 1. Mapa de ubicación de puntos de monitoreo. 


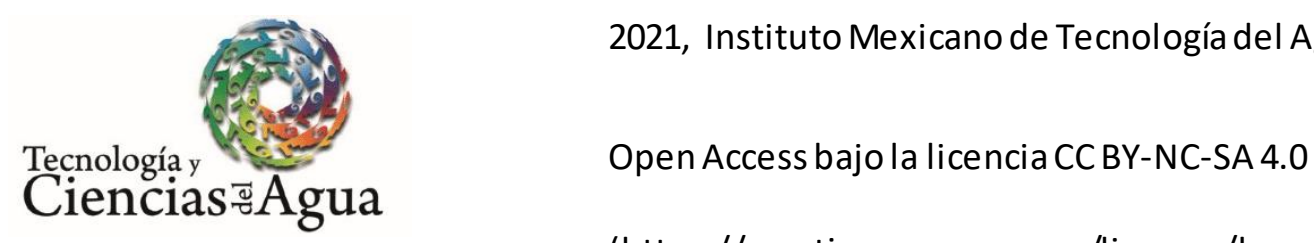

(https://creativecommons.org/licenses/by-nc-sa/4.0/)

Tabla 1. Coordenadas de ubicación de los puntos de muestreo.

\begin{tabular}{|c|c|c|c|c|c|c|c|c|c|c|c|c|}
\hline \multirow[b]{2}{*}{$x$} & \multirow[b]{2}{*}{$r$} & \multirow[b]{2}{*}{ Descripción } & \multirow[b]{2}{*}{ Zona } & \multirow{2}{*}{ Ramal } & \multirow{2}{*}{$\begin{array}{c}\text { Altura } \\
\text { (msnm) }\end{array}$} & \multirow[b]{2}{*}{ Parroquia } & \multirow{2}{*}{$\begin{array}{l}\text { Características } \\
\text { de cobertura } \\
\text { del suelo }\end{array}$} & \multirow{2}{*}{$\begin{array}{c}\text { Pendiente } \\
(\%)\end{array}$} & \multirow{2}{*}{\begin{tabular}{|c|} 
Longitud \\
del \\
cauce \\
del \\
tramo
\end{tabular}} & \multirow{2}{*}{\begin{tabular}{|c|} 
Longitud \\
del \\
cauce \\
desde la \\
naciente
\end{tabular}} & \multicolumn{2}{|c|}{ Gasto base $\left(\mathrm{m}^{3} / \mathrm{s}\right)$} \\
\hline & & & & & & & & & & & Normal & $\begin{array}{c}\text { Baja } \\
\text { precipitación }\end{array}$ \\
\hline \multicolumn{13}{|c|}{ Curso principal } \\
\hline 160336 & 9841315 & Cabeceras del río Puyo* & Alta-rural & Puyo & 1256 & Mera & $\overline{B S}$ & 7.0 & 0 & 0 & 0.10 & 0.09 \\
\hline 165474 & 9841913 & Dique de Fátima* & \begin{tabular}{|l|} 
Alta-rural \\
\end{tabular} & Puyo & 996 & Fátima & $B S+P$ & 5.2 & 8 & 8 & 0.86 & 0.71 \\
\hline 166665 & 9835765 & Barrio La Isla* & Urbana & Puyo & 926 & Puyo & $\overline{Z U}$ & 1.1 & 9 & 17 & 5.00 & 3.72 \\
\hline 169329 & 9830911 & Puente de Unión Base & \begin{tabular}{|l|} 
Baja-rural \\
\end{tabular} & Puyo & 901 & Pomona & $\mathrm{BS}+\mathrm{P}$ & 0.5 & 7 & 24 & 7.42 & 5.63 \\
\hline 157105 & 9838531 & Estación Biológica Pindo Mirador & Alta-rural & Pindo & 1134 & Shell & $\overline{B S}+P$ & 7.5 & 4.2 & 4.2 & 0.76 & 0.37 \\
\hline 159382 & 9834582 & Dique de Shell* & Urbana & Pindo & 1045 & Shell & $B S+P$ & 1.8 & 5 & 9.2 & 2.63 & 1.45 \\
\hline 164881 & 9834360 & Camal Municipal & Urbana & Pindo & 921 & Puyo & $\overline{Z U}$ & 2.5 & 8 & 17.2 & 6.03 & 1.63 \\
\hline 169595 & 9831059 & Puente A Ilupungu & Baja-rural & Puyo & 901 & Puyo & $\mathrm{BS}+\mathrm{P}+\mathrm{ZU}$ & 0.3 & 7.8 & 25 & 6.95 & 3.19 \\
\hline 171825 & 9828556 & Rosario Yacu & Baja-rural & Puyo & 887 & Veracruz & $B S+P+C$ & 0.2 & 5.2 & 30.2 & 18.32 & 13.77 \\
\hline 174914 & 9818079 & Bellavista Baja & Baja-rural & Puyo & 801 & Madre & $B S+P$ & 0.9 & 15.7 & 45.9 & 27.61 & 24.01 \\
\hline \multicolumn{13}{|c|}{ Afluentes } \\
\hline$x$ & $Y$ & \multicolumn{2}{|l|}{ Descripción del punto } & Zona & Ramal & \begin{tabular}{|c|c|} 
Altura \\
$($ msnmm)
\end{tabular} & Parroquia & Caracterís & $\begin{array}{l}\text { isticas de co } \\
\text { del suelo }\end{array}$ & bertura & Gasto & pase $\left(m^{3} / s\right)$ \\
\hline 177246 & 9828776 & Puente sobre el río Chorre & eras & Baja-rural & Puyo & 992 & Veracruz & & $B S+P+C$ & & 0.61 & 0.37 \\
\hline 174498 & 9834557 & Cabeceras del río Sandal & & Alta-rural & Puyo & 983 & Veracruz & & $\overline{B S}+P+C$ & & 0.04 & 0.03 \\
\hline 173746 & 9837726 & Dique de Los Mecánicos* (río C & hilcayacu) & Alta-rural & Puyo & 979 & Veracruz & & $B S+P+C$ & & 0.09 & 0.06 \\
\hline 173397 & 9834595 & Dique de Veracruz* (río San & dalias) & Alta-rural & Puyo & 959 & Veracruz & & $\overline{B S}+P+C$ & & 0.33 & 0.10 \\
\hline 165582 & 9837248 & Dique Pambay* (río Pamb & ay) & Alta-urbana & Puyo & 952 & Puyo & & $\overline{B S}+P+Z U$ & & ND & ND \\
\hline 168291 & 9834451 & Paico (río Chilcayacu) & & Urbana & Puyo & 923 & Puyo & & $\overline{B S}+P+Z U$ & & 0.70 & ND \\
\hline 163223 & 9833715 & Dique Las Palmas* & & Urbana & Pindo & 966 & Puyo & & $\mathrm{BS}+\mathrm{ZU}$ & & ND & ND \\
\hline 170644 & 9833900 & Puente del río Sandalia & & Urbana & Puyo & 953 & Puyo & & $\overline{B S}+P+Z U$ & & 1.17 & 0.80 \\
\hline 170727 & 9828064 & Comunidad Dos Ríos (río Chin & gushimi) & Baja-rural & Puyo & 906 & Veracruz & & $\overline{B S}+P+C$ & & 1.92 & $\overline{N D}$ \\
\hline 175231 & 9826044 & Mina Chorreras & & Baja-rural & Puyo & 860 & Veracruz & & $B S+P+C$ & & 1.16 & 0.65 \\
\hline 180945 & 9824657 & Puente sobre el río Indula & $\mathrm{ma}$ & Baja-rural & Puyo & 998 & Pomona & & $\overline{B S}+P+C$ & & 0.42 & 0.23 \\
\hline 176956 & 9824201 & Balneario del río Indulam & & Baja-rural & Puyo & 998 & Pomona & & $\mathrm{BS}+\mathrm{P}+\mathrm{C}$ & & 1.74 & 1.23 \\
\hline 164396 & 9827279 & Cabeceras del río Putuim & & Baja-rural & Puyo & 937 & Madre Tierra & & $B S+P+C$ & & 0.28 & 0.05 \\
\hline 170229 & 9821720 & Nueva Vida & & Baja-rural & Puyo & 833 & Tarqui & & $B S+P+C$ & & 1.80 & 1.20 \\
\hline 174838 & 9818474 & Bellavista & & Baja-rural & Puyo & 799 & Madre Tierra & & $B S+P+C$ & & 4.19 & 1.76 \\
\hline 169686 & 9832099 & Puente de Ila Yacu & & Baja-rural & Puyo & 921 & Veracruz & & $\mathrm{BS}+\mathrm{P}$ & & 2.48 & $\mathrm{ND}$ \\
\hline 164592 & 9830277 & Cabeceras río Chingushir & & Baja-rural & Puyo & 941 & Tarqui & & $B S+P+C$ & & 0.06 & 0.05 \\
\hline
\end{tabular}

*Puntos también considerados en prueba piloto BS: bosque secundario; P: pastizal, C:

cultivo; ZH: zona urbana: ND: dato no disponible.

Se agruparon los puntos de monitoreo en tres zonas, considerándose como zona alta aquella ubicada en la cuenca alta con pendientes $\geq 5 \%$ y donde no existen asentamientos poblacionales urbanos; zona urbana, 


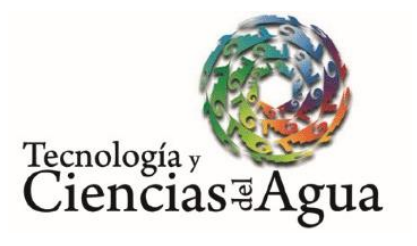

2021, Instituto Mexicano de Tecnología del Agua

Open Access bajo la licencia CC BY-NC-SA 4.0

(https://creativecommons.org/licenses/by-nc-sa/4.0/)

aquella con asentamientos poblacionales superiores a 1000 personas; y zona baja, aquella ubicada aguas abajo posterior a los asentamientos poblacionales. También se registró para referencia las precipitaciones diarias y mensuales durante el periodo de estudio con el uso de una estación meteorológica automática en el punto Estación Biológica Pindo Mirador, en la zona alta. Con los resultados obtenidos se aplicó una correlación de Pearson (Fernández \& Díaz, 2001) con el uso del programa SPSS (IBM, 2013), para determinar en función de cada zona la relación existente entre los diferentes parámetros evaluados, donde también se incluyeron las variables de la precipitación presente el día de monitoreo y la precipitación del día anterior.

Se determinó el estadístico coeficiente de variación desde los puntos de monitoreo y se realizó un análisis de varianza con pruebas de comparación de Duncan para los diferentes parámetros en función de las zonas, para lo cual se utilizó el programa estadístico INFOSTAT (Di-Rienzo et al., 2014). Previo al análisis, los valores de porcentaje de saturación de oxígeno se transformaron con la función raíz cuadrada (Herrera, 2013). Los valores de los diferentes parámetros se compararon con la normativa ecuatoriana referida en el Texto Unificado de Legislación Ambiental Secundaria (TULSMA), Libro VI Anexo 1 (Ministerio del Ambiente del Ecuador, 2015), considerando los criterios de calidad de agua para uso recreativo con contacto primario, y criterios de calidad admisibles para la preservación de la vida acuática y silvestre en aguas dulces, marinas y de estuarios.

Se aplicó una encuesta en las poblaciones cercanas al curso principal y sus afluentes, para identificar los tipos de actividades productivas que se realizan y la disponibilidad de servicios básicos, que fueron distribuidos en función de la densidad poblacional de estos sectores; la encuesta se aplicó 


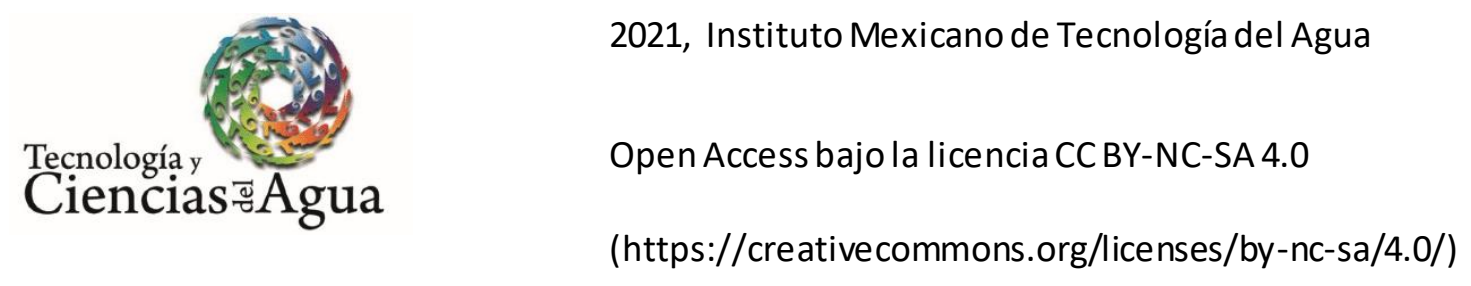

a un total de 341 pobladores ( 25 en la zona alta, 212 en la urbana y 104 en la baja) y a 72 en los afluentes (cinco en la zona alta y 67 en la baja), dando un total de 413 cuestionarios, cuyo número supera el valor calculado para el tamaño de muestra de poblaciones finitas (Ecuación (1)), que es de 165, con un nivel de confianza del $99 \%$ (Morales, 2011), considerando una población de 60130 habitantes.

Tamaño de muestra:

$\mathrm{n}=\frac{\mathrm{NZ}^{2} \mathrm{pq}}{(\mathrm{N}-1) \mathrm{e}^{2}+\mathrm{Z}^{2} \mathrm{pq}}$

Donde $n$ es el tamaño de la muestra; $N$, tamaño del universo (total población); $Z$, nivel de confianza de la estimación, considerando el $99 \%$ de confianza (2.576); $p$, probabilidad de aceptación (0.5); $q$, probabilidad de rechazo (0.5), y e que es el margen de error (10\%).

\section{Resultados}

La Figura 2 permite observar las características de precipitación recolectadas en la estación meteorológica automática ubicada en la estación Pindo Mirador, en la zona de estudio en el periodo de muestreo, con precipitaciones anuales con una media de $5005 \mathrm{~mm}$ y un promedio mensual 


\section{1, Instituto Mexicano de Tecnología del Agua \\ Tecnología y \\ Ciencias $\underset{\mho}{\mho}$ gua \\ Open Access bajo la licencia CCBY-NC-SA 4.0 \\ (https://creativecommons.org/licenses/by-nc-sa/4.0/)}

de $417 \mathrm{~mm}$, donde sus meses menos Iluviosos presentan precipitaciones superiores a los $190 \mathrm{~mm}$; se tienen como épocas de mayor precipitación los meses de abril de 2015 y enero de 2017; y de menor precipitación septiembre de 2015, enero de 2016 y febrero de 2018. Las características de precipitación reportadas para los días de muestreo y día anterior fueron en 2015 en los muestreos de febrero: 9 y $11.8 \mathrm{~mm}$; marzo: 0 y $2.2 \mathrm{~mm}$; abril: 17.6 y $4.4 \mathrm{~mm}$; junio: 0.2 y $4.4 \mathrm{~mm}$. En 2016, en noviembre: 16.4 y $18 \mathrm{~mm}$; diciembre: 5.6 y $0 \mathrm{~mm}$. En 2017, en septiembre: 3.7 y $8.2 \mathrm{~mm}$; noviembre: 12.2 y $9.8 \mathrm{~mm}$. En 2018, en septiembre: 7.3 y $14.2 \mathrm{~mm}$, y 9.9 y $9.3 \mathrm{~mm}$, y en octubre: 4.7 y $8.4 \mathrm{~mm}$.

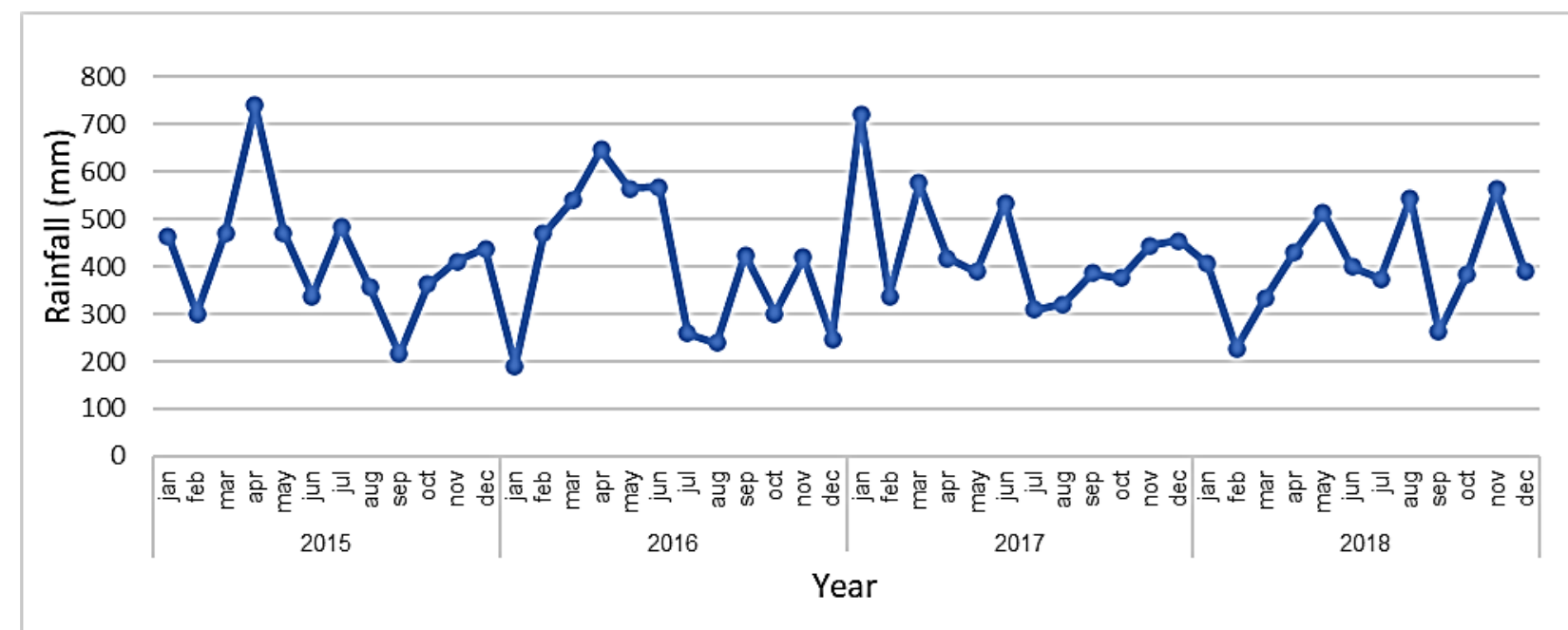

Figura 2. Precipitaciones mensuales registradas en la estación biológica Pindo Mirador, localizada en el área de estudio. Fuente: datos colectados de la estación meteorológica automática.

La conductividad eléctrica a nivel general en todas las fechas (Figura 3) presenta mayores reportes en el curso principal, en la zona alta, en los diques Fátima, Pambay y las Palmas, donde su media sobrepasa los 100 
Tecnología y

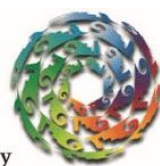

Ciencias $\stackrel{\Xi}{\triangleleft}$ gua
2021, Instituto Mexicano de Tecnología del Agua

Open Access bajo la licencia CCBY-NC-SA 4.0

(https://creativecommons.org/licenses/by-nc-sa/4.0/)

$\mathrm{uS} / \mathrm{cm}^{2}$, posteriormente disminuye en las zonas bajas de la cuenca; esto puede deberse a la presencia de mayores caudales, que generan mayor dilución. En los afluentes se reporta mayor conductividad eléctrica en la zona alta en el dique Pambay, así como en la zona urbana, en el dique Las Palmas; los valores son menores en los otros puntos de monitoreo. El punto dique de Shell es el que reporta mayor coeficiente de variación (Tabla 2).

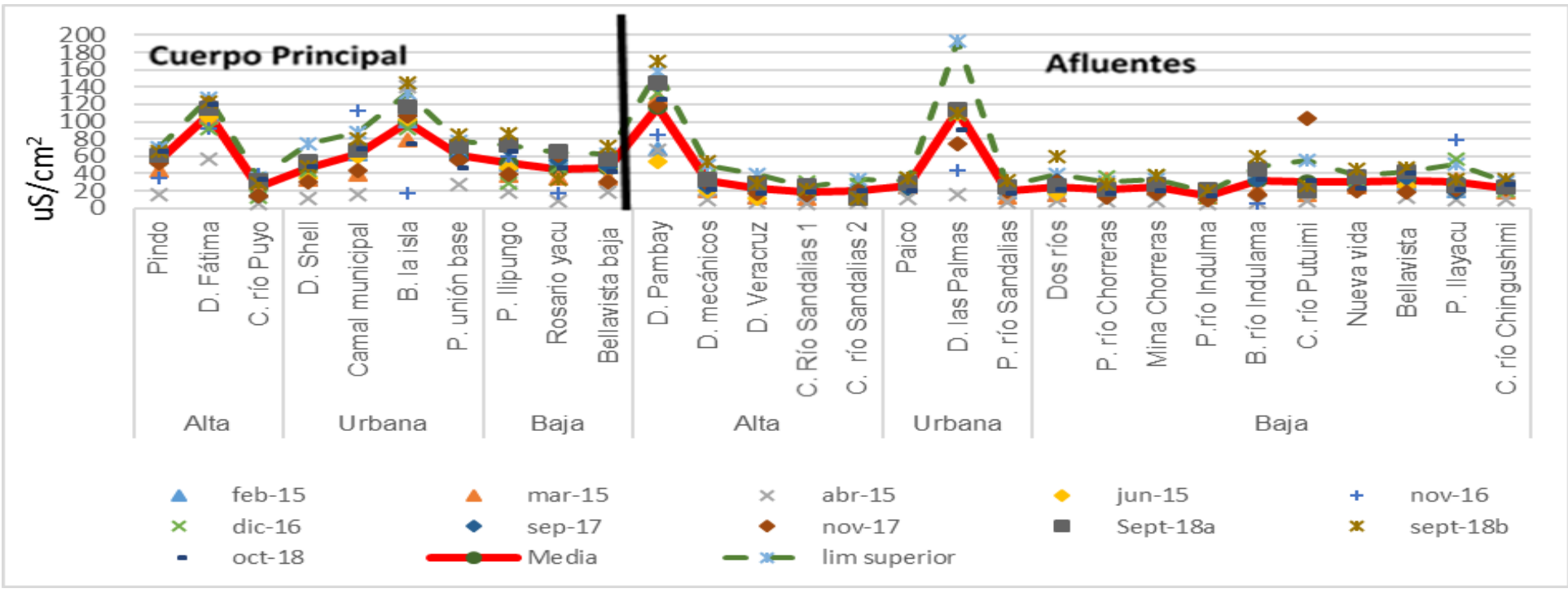

Figura 3. Valores registrados de conductividad.

Tabla 2. Coeficiente de variación (CV) de las variables registradas.

\begin{tabular}{|l|c|c|c|c|c|c|c|}
\hline \multicolumn{1}{|c|}{ Punto } & $\begin{array}{c}\text { Sat. de } \\
\text { oxígeno }\end{array}$ & $\begin{array}{c}\text { Colif. } \\
\text { totales }\end{array}$ & $\begin{array}{c}\text { Colif. } \\
\text { fecales }\end{array}$ & $\begin{array}{c}\text { Conduc- } \\
\text { tividad }\end{array}$ & pH & DQO & DBO5 \\
\hline $\begin{array}{l}\text { E. B. Pindo } \\
\text { Mirador }\end{array}$ & 17.8 & 162.1 & 148.6 & 29.6 & 4.8 & 57.3 & 96.5 \\
\hline $\begin{array}{l}\text { Dique de } \\
\text { Fátima }\end{array}$ & 17.8 & 162.0 & 235.3 & 18.4 & 5.3 & 66.4 & 83.0 \\
\hline
\end{tabular}




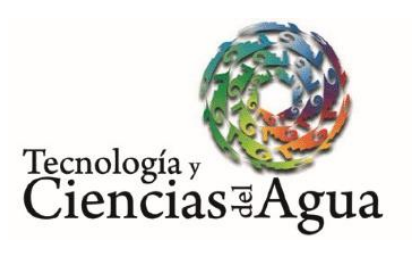

2021, Instituto Mexicano de Tecnología del Agua

Open Access bajo la licencia CCBY-NC-SA 4.0

(https://creativecommons.org/licenses/by-nc-sa/4.0/)

\begin{tabular}{|c|c|c|c|c|c|c|c|}
\hline $\begin{array}{l}\text { Cabecera del } \\
\text { río Puyo }\end{array}$ & 25.7 & 241.7 & 225.9 & 45.8 & 11.2 & 57.7 & 103.2 \\
\hline Dique de Shell & 24.6 & 100.6 & 123.7 & 31.3 & 6.2 & 72.9 & 101.2 \\
\hline $\begin{array}{l}\text { Camal } \\
\text { municipal }\end{array}$ & 24.9 & 129.0 & 88.1 & 39.8 & 7.6 & 60.5 & 72.1 \\
\hline Barrio La Isla & 18.0 & 106.3 & 141.6 & 35.3 & 3.7 & 62.7 & 92.2 \\
\hline $\begin{array}{l}\text { Puente Unión } \\
\text { Base }\end{array}$ & 10.3 & 136.7 & 250.9 & 25.2 & 7.4 & 46.8 & 92.0 \\
\hline $\begin{array}{l}\text { Puente } \\
\text { Ilipungo }\end{array}$ & 20.1 & 186.6 & 111.6 & 39.0 & 7.2 & 62.5 & 103.0 \\
\hline $\begin{array}{l}\text { Rosario } \\
\text { Yacu }\end{array}$ & 13.2 & 149.4 & 148.0 & 42.4 & 7.7 & 58.5 & 79.2 \\
\hline Bellavista baja & 20.8 & 159.7 & 200.0 & 32.0 & 8.6 & 87.1 & 79.7 \\
\hline Dique Pambay & 22.6 & 178.8 & 94.5 & 33.5 & 3.4 & 119.1 & 132.4 \\
\hline $\begin{array}{l}\text { Dique Los } \\
\text { Mecánicos }\end{array}$ & 22.7 & 113.6 & 198.6 & 43.5 & 10.0 & 56.5 & 93.0 \\
\hline $\begin{array}{l}\text { Dique } \\
\text { Veracruz }\end{array}$ & 24.9 & 201.7 & 112.6 & 41.6 & 8.8 & 71.8 & 120.7 \\
\hline $\begin{array}{l}\text { C. río } \\
\text { Sandalias } 1\end{array}$ & 27.3 & 274.0 & 188.1 & 36.1 & 7.6 & 45.0 & 127.4 \\
\hline $\begin{array}{l}\text { C. río } \\
\text { Sandalias } 2\end{array}$ & 27.2 & 115.9 & 150.8 & 37.0 & 9.1 & 64.3 & 118.3 \\
\hline Paico & 23.2 & 232.5 & 228.5 & 27.0 & 7.0 & 76.1 & 102.4 \\
\hline $\begin{array}{l}\text { Dique Las } \\
\text { Palmas }\end{array}$ & 25.5 & 214.1 & 130.5 & 36.6 & 3.5 & 79.1 & 101.3 \\
\hline $\begin{array}{l}\text { Puente del río } \\
\text { Sandalias }\end{array}$ & 8.8 & 211.1 & 124.3 & 32.2 & 8.7 & 94.4 & 153.1 \\
\hline Dos ríos & 22.9 & 189.0 & 184.9 & 56.2 & 8.8 & 73.0 & 92.0 \\
\hline
\end{tabular}




\section{1, Instituto Mexicano de Tecnología del Agua
Tecnología y
Ciencias $\cong$ Open Access bajo la licencia CC BY-NC-SA 4.0
(https://creativecommons.org/licenses/by-nc-sa/4.0/)}

\begin{tabular}{|l|c|c|c|c|c|c|c|}
\hline $\begin{array}{l}\text { Puente del río } \\
\text { Chorreras }\end{array}$ & 21.6 & 240.8 & 132.7 & 40.6 & $\mathbf{1 1 . 1}$ & 38.5 & 103.1 \\
\hline $\begin{array}{l}\text { Mina } \\
\text { Chorreras }\end{array}$ & 14.4 & 259.6 & 159.4 & 34.9 & 9.2 & 46.9 & 119.5 \\
\hline $\begin{array}{l}\text { Puente del río } \\
\text { Indulama }\end{array}$ & 17.8 & 203.7 & 208.3 & 32.8 & 10.9 & 67.2 & 115.5 \\
\hline $\begin{array}{l}\text { Balneario río } \\
\text { Indulama }\end{array}$ & 24.2 & $\mathbf{2 8 7 . 3}$ & 156.3 & 54.7 & 8.5 & 69.1 & 85.4 \\
\hline C. río Putuimi & 26.7 & 256.0 & 217.3 & $\mathbf{9 0 . 1}$ & 10.4 & 45.2 & 87.2 \\
\hline Nueva vida & 18.2 & 158.8 & 152.3 & 26.2 & 8.7 & 54.3 & 81.5 \\
\hline Bellavista & $\mathbf{2 7 . 4}$ & 200.5 & 185.9 & 31.7 & 7.5 & 84.0 & 117.7 \\
\hline $\begin{array}{l}\text { Puente del río } \\
\text { Ilayacu }\end{array}$ & 17.8 & 166.5 & 149.5 & 65.2 & 7.2 & 48.1 & 93.6 \\
\hline $\begin{array}{l}\text { C. río } \\
\text { Chingushimi }\end{array}$ & 19.8 & 143.3 & 172.7 & 26.6 & 9.2 & 37.8 & 75.7 \\
\hline Media & 20.92 & 185.04 & 165.03 & 38.76 & 7.83 & 64.38 & 100.77 \\
\hline
\end{tabular}

El potencial hidrógeno (Figura 4a) presentó valores entre 6 y 9 en los puntos de monitoreo en el curso principal; se compararon los valores con los criterios de calidad admisibles para la preservación de la vida acuática y silvestre en aguas dulces que establece valores entre 6.5 y 9, y para criterios de calidad admisibles para uso recreativo con contacto primario con valores entre 6.5 y 8.3 (Ministerio del Ambiente del Ecuador, 2015). La mayoría de puntos presentan valores debajo del límite permisible en junio de 2015; en abril de 2015 ocurre lo mismo en los puntos Bellavista Bajo, Cabecera del río Sandalias 2, puente sobre el río Chorreras, puente sobre el río Indulama, balneario del río Indulama, puente Ilayacu y cabeceras del 
Ciencias $\stackrel{\text { Tecnolóa }}{\text { Cien }}$

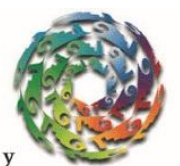

2021, Instituto Mexicano de Tecnología del Agua

Open Access bajo la licencia CCBY-NC-SA 4.0

(https://creativecommons.org/licenses/by-nc-sa/4.0/)

río Chingushimi; en noviembre de 2017 se registró el valor más bajo de todos en las cabeceras del río Sandalias 2.

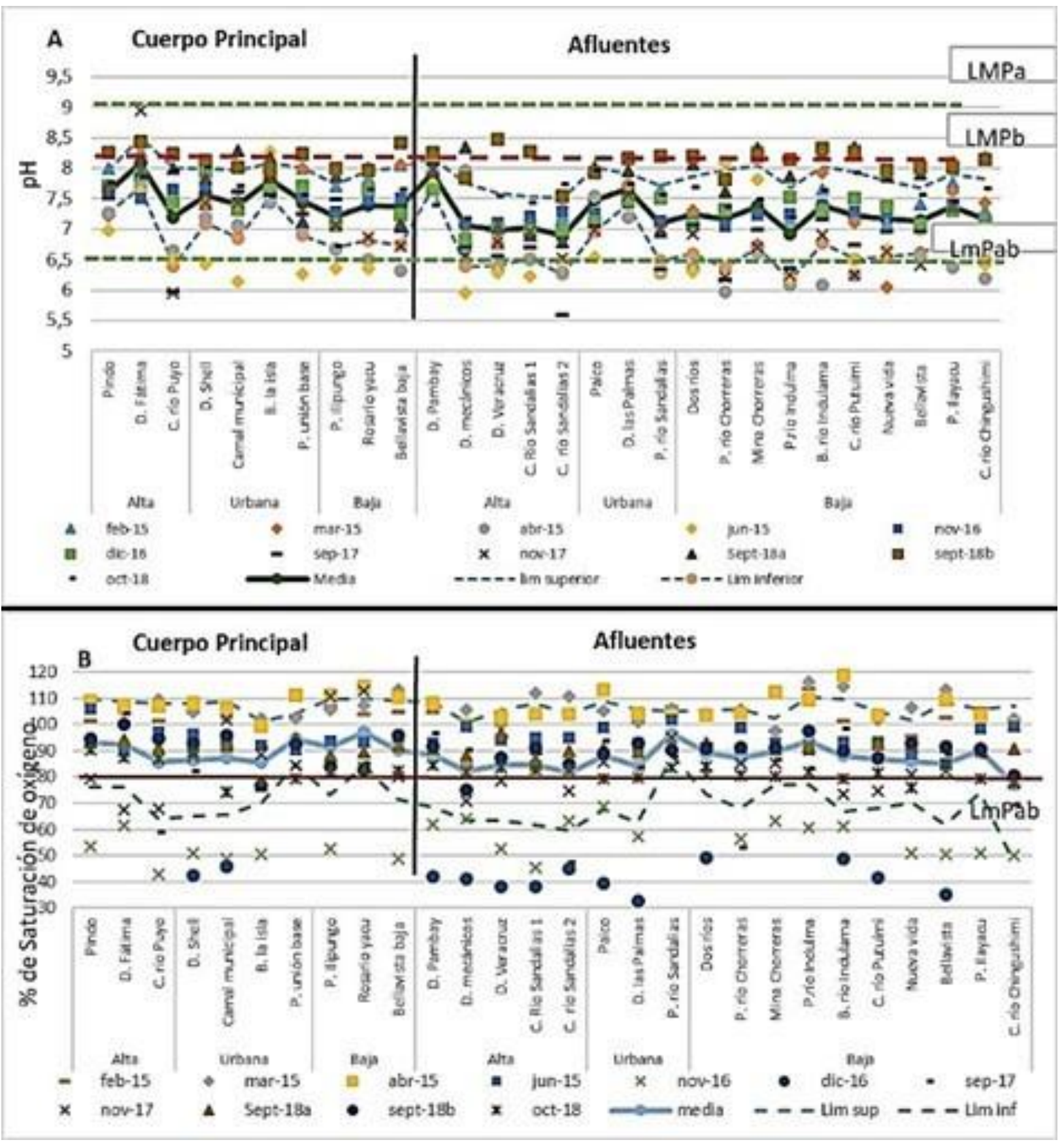

Figura 4. Valores registrados de: a) potencial hidrógeno; b) saturación de oxígeno. 


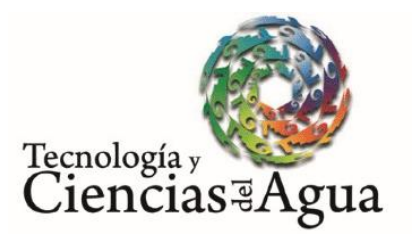

2021, Instituto Mexicano de Tecnología del Agua

Open Access bajo la licencia CCBY-NC-SA 4.0

(https://creativecommons.org/licenses/by-nc-sa/4.0/)

El límite máximo permisible sobre criterios de calidad de agua para preservación de la vida acuática y silvestre en aguas dulces no fue sobrepasado en los puntos y fechas de muestreo; pero en el criterio de uso recreativo con contacto primario, en el cuerpo principal el balnearios dique de Fátima sobrepasa el límite en noviembre de 2017 y septiembre de 2018(b); en los afluentes, los balnearios Dique de Veracruz y balneario del río Indulama superan el límite permisible en septiembre de 2018(b). La media general muestra los valores más altos en los diques de Fátima y Pambay, los cuales se encuentran cercanos al límite.

Los niveles de saturación de oxígeno (Figura 4b) estuvieron en todos los puntos sobre el límite mínimo permisible, que para ambos criterios señalados debe sobrepasar el 80 \% de saturación, con excepción de las fechas de junio de 2015 en los diques Shell, Los Mecánicos, Veracruz; Las Palmas, en los puntos Camal Municipal, Cabeceras del río Sandalias 1 y 2, Dos Ríos, balneario del río Indulama, cabeceras del río Putuimi y Bellavista; también algunos puntos en noviembre de 2016 y octubre de 2018. Los puntos donde reiteradamente se reportan valores por debajo del límite mínimo son en el cuerpo principal de las cabeceras del río Puyo, el Camal Municipal, barrio La Isla, y en los afluentes de los diques Los Mecánicos y Veracruz, y en las cabeceras del río Sandalias 2. Los promedios del curso principal registran valores superiores al $80 \%$, disminuyendo en la zona urbana e incrementándose en la zona baja, lo cual, al ser el mismo curso hídrico, representan una reoxigenación en las zonas bajas. En los afluentes, las menores concentraciones se tuvieron en el dique Mecánicos, y en las cabeceras del río Sandalias 2.

La demanda química de oxígeno (Figura $5 a$ ) reportó valores más altos en los meses de febrero y abril del 2015, principalmente en los sectores de 


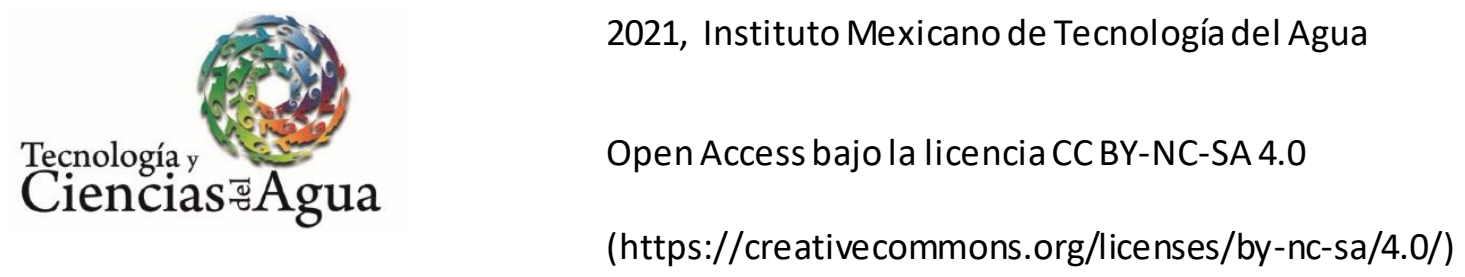

Bellavista Baja, dique de Fátima y barrio La Isla en el curso principal, y en los puntos dique de Veracruz, cabeceras del río Sandalias 2, puente sobre rio Indulama, balneario sobre el río Indulama y Bellavista en los afluentes; y en septiembre de 2018, en el dique Pambay. Los promedios por punto muestran mayores valores en el barrio La Isla y Bellavista; baja en el curso principal y en las cabeceras del río Sandalias 2, Bellavista y cabeceras del río Chingushimi en los afluentes. Este parámetro no está contemplado en la normativa legal ecuatoriana, por lo cual no se compara con límites permisibles. 
Ciencias Cigua

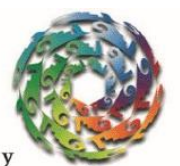

2021, Instituto Mexicano de Tecnología del Agua

Open Access bajo la licencia CCBY-NC-SA 4.0

(https://creativecommons.org/licenses/by-nc-sa/4.0/)
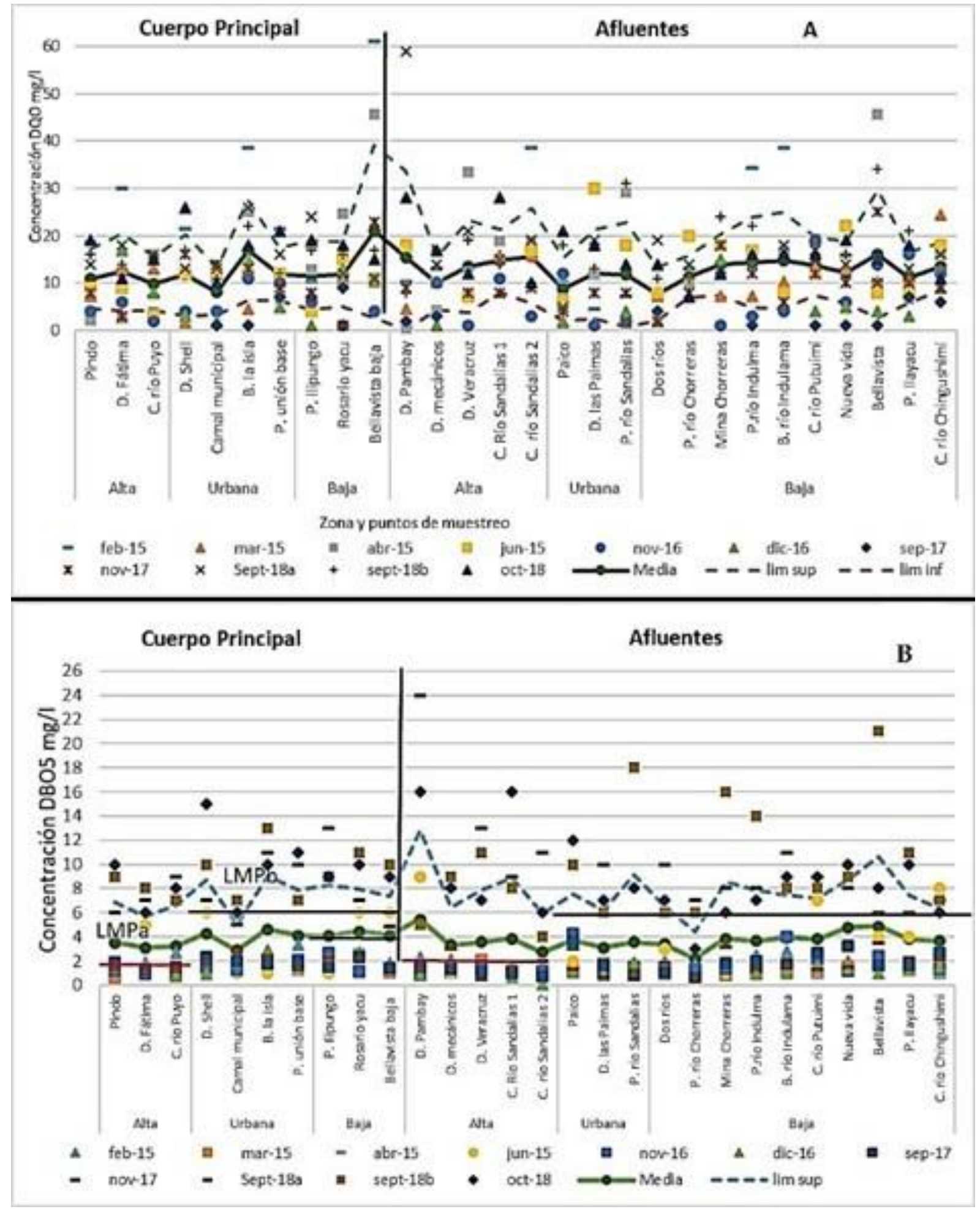

Figura 5. Valores registrados de concentraciones de: a) demanda química de oxígeno; b) demanda bioquímica de oxígeno. 


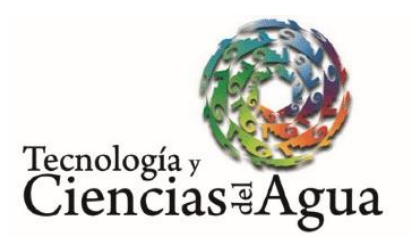

2021, Instituto Mexicano de Tecnología del Agua

Open Access bajo la licencia CCBY-NC-SA 4.0

(https://creativecommons.org/licenses/by-nc-sa/4.0/)

En la demanda bioquímica de oxígeno (figura $5 b$ ) se consideró el límite máximo permisible para criterios de calidad admisible de la $\mathrm{DBO}_{5}$ para la protección de la vida acuática no impactada, que corresponde a zonas de la cuenca alta, donde el límite es $2 \mathrm{mg} / \mathrm{l}$, y con impacto moderado correspondiente a zonas urbana y baja, donde el límite máximo es $6 \mathrm{mg} / \mathrm{l}$. Los muestreos indicaron valores superiores a los establecido en la norma en el curso principal en 2015 en la zona alta en febrero en las cabeceras del río Puyo; en el mes de abril, en el punto Pindo; mientras en el mes de junio, en la zona alta, en el punto dique de Fátima y en los afluentes en el mes de junio, en la zona alta en el dique del río Pambay; y en la zona baja, en las cabeceras del río Putuimi, Nueva Vida y cabeceras del río Chingushimi. En los muestreos de noviembre de 2017, septiembre de 2018 ( $a$ y b), y octubre de 2018, la mayoría de puntos sobrepasa el límite máximo permisible. Los valores promedio en el curso principal y afluentes se encuentran dentro de lo establecido en los límites permisibles de la normativa ecuatoriana en las zonas urbana y baja, donde se considera una condición de impacto moderado para la vida acuática; pero no así en la zona alta, donde se considera vida acuática no impactada. A su vez, se reportan mayores concentraciones en el curso principal en los sectores Rosario Yacu, dique de Shell y barrio La Isla, y en los afluentes en el dique Pambay.

En los valores de coliformes totales (Figura 6a), el criterio de calidad de agua para uso recreativo con contacto primario establece un límite máximo permisible de 2000 en su número más probable/100 ml. En lo general, todos los puntos sobrepasan el límite máximo permisible, excepto las fechas abril de 2015; septiembre de 2018 (a y b); y octubre de 2018, donde la mayoría de puntos presenta valores debajo del límite permisible. La media general muestra que sólo el punto dique de Los Mecánicos alcanza 


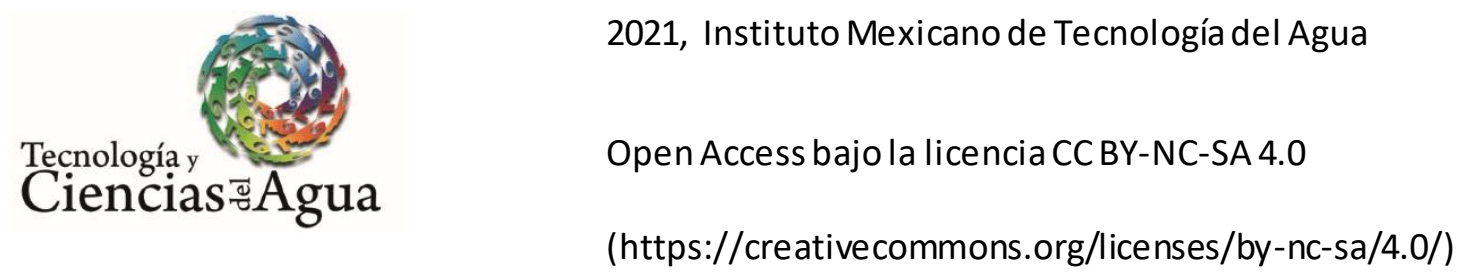

el límite máximo permisible. En el cuerpo principal se identifica que la zona alta presenta menores concentraciones, y éstas se incrementan a partir del paso del curso hídrico por la zona urbana; en los afluentes, al ser cada uno un curso independiente, no se muestra esta tendencia. En la medida de coliformes fecales (Figura 6b), el criterio de calidad de agua para uso recreativo con contacto primario establece un límite maximo de 200 NMP/100 ml. El curso principal presenta valores dentro de los límites; lo mismo ocurre en la zona alta, los puntos Pindo y dique de Fátima en los meses de marzo y junio de 2015, diciembre de 2016 septiembre y noviembre de 2017; así como en la zona urbana, en el punto dique de Shell, en los meses de marzo y abril. 


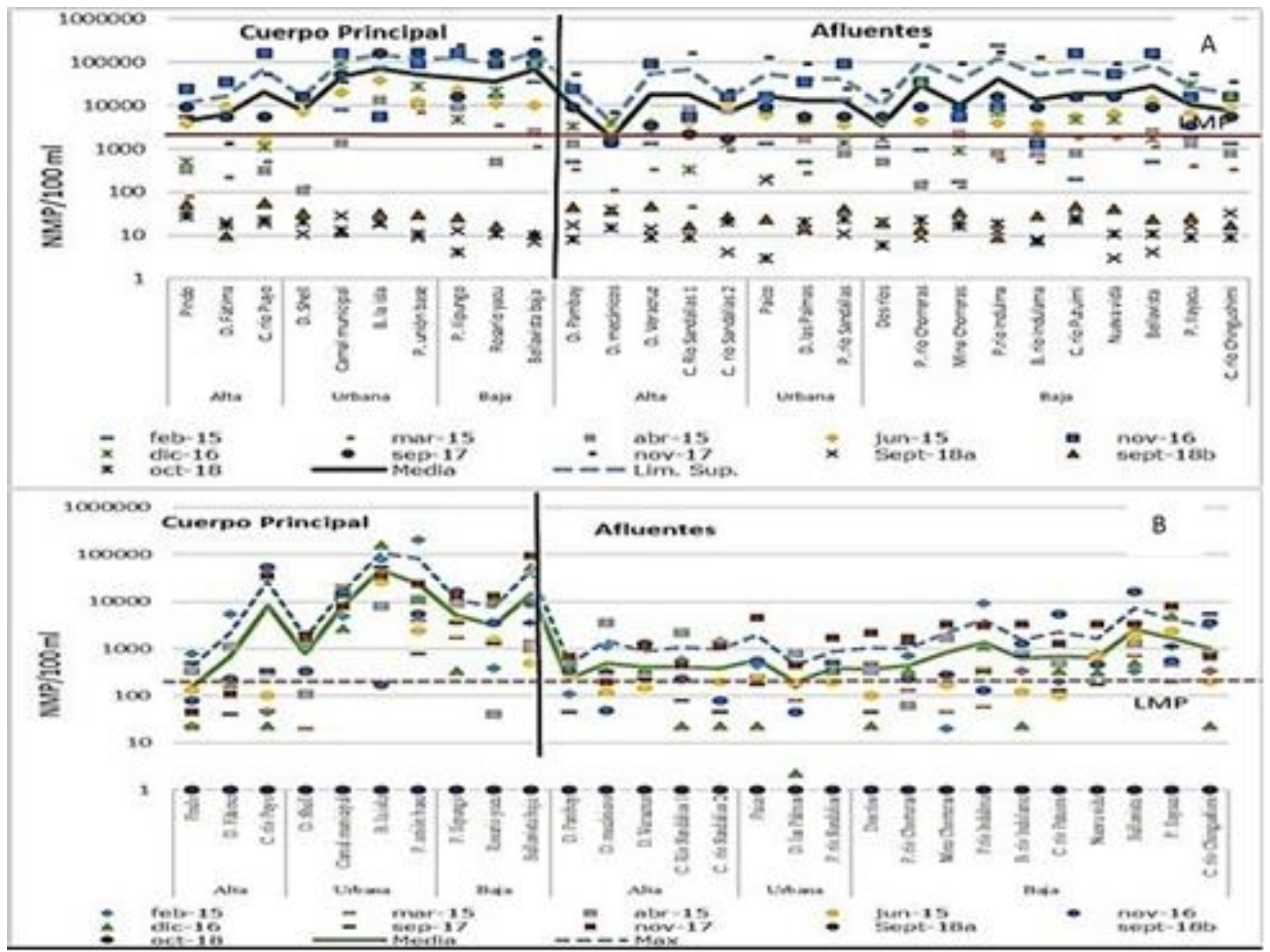

Figura 6. Concentración de: a) coliformes totales; b) coliformes fecales.

A partir de este punto se incrementa de forma logarítmica la concentración de coliformes fecales en la zona urbana y posteriormente disminuye en la zona baja, donde se mantiene sobre el límite máximo permisible. Los afluentes, en los meses de marzo y junio de 2015, y diciembre de 2016, presentan valores debajo del límite máximo permisible. Los valores medios muestran a nivel general valores dentro del límite permisible en el cuerpo principal en el punto Pindo y en los afluentes en el dique "Las Palmas". El resto de puntos sobrepasa los límites permisibles, 


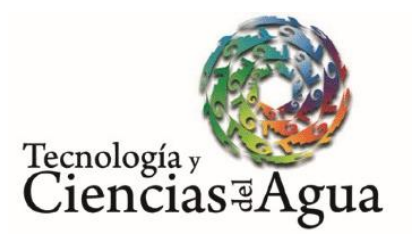

2021, Instituto Mexicano de Tecnología del Agua

Open Access bajo la licencia CC BY-NC-SA 4.0

(https://creativecommons.org/licenses/by-nc-sa/4.0/)

teniéndose las mayores concentraciones en el barrio La Isla, el puente de Unión Base y Bellavista baja. Los muestreos realizados en 2018 septiembre (a y b), y octubre reportaron valores de 1 NMP.

En la Tabla 2 se muestran los coeficientes de variación para cada parámetro en cada punto, donde se puede apreciar a nivel general que las variables con mayores coeficientes de variación son coliformes totales y fecales, y demanda bioquímica de oxígeno; a su vez, para cada parámetro tenemos los puntos con mayor coeficiente de variación en cuanto a saturación de oxígeno y coliformes totales en cabeceras del río Sandalias 1; coliformes fecales, en el puente de Unión Base; conductividad, en las cabeceras del río Putuimi; potencial hidrógeno, en las cabeceras del río Puyo; demanda química de oxígeno, en el dique del río Pambay; y demanda biológica de oxígeno, en el Puente del río Sandalias.

El análisis de varianza entre zonas (Tabla 3) reportó diferencias al 0.05 entre zonas para los parámetros conductividad eléctrica, y coliformes totales en el curso principal; mientras que en los afluentes se reportó para conductividad eléctrica y coliformes fecales. La prueba de comparación de medias de Duncan (Tabla 3) mostró que en la zona baja la conductividad eléctrica en el curso principal presenta diferencias con la zona alta y urbana, reportando los valores más bajos de las tres zonas, lo cual puede deberse a efectos de dilución por aumento de los volúmenes de agua en este sector. Este comportamiento también se observa en los afluentes. En el curso principal, la medida de coliformes totales en la zona baja presenta diferencias con respecto a las otras zonas. En los afluentes, la concentración de coliformes fecales reporta mayores valores en la zona baja. En ambos casos puede deberse a efectos de acumulación de materiales depositados en las aguas residuales colectadas en las zonas alta y urbana. 
Tabla 3. Análisis de varianza y prueba de comparación de medias de los parámetros evaluados entre zonas para el curso principal y afluentes.

\begin{tabular}{|c|c|c|c|c|}
\hline \multirow{2}{*}{\multicolumn{3}{|c|}{ Parámetro }} & \multicolumn{2}{|c|}{ Valor de $\mathbf{P}$} \\
\hline & & & \begin{tabular}{|l} 
Curso principal \\
\end{tabular} & Afluentes \\
\hline \multicolumn{3}{|c|}{ Conductividad } & 0.0385 & 0.001 \\
\hline \multicolumn{3}{|c|}{ Saturación de oxígeno } & 0.5834 & 0.4739 \\
\hline \multicolumn{3}{|c|}{$\mathrm{pH}$} & 0.0742 & 0.2309 \\
\hline \multicolumn{3}{|c|}{ Demanda química de oxígeno } & 0.3074 & 0.343 \\
\hline \multicolumn{3}{|c|}{ Demanda bioquímica de oxígeno } & 0.5939 & 0.8955 \\
\hline \multicolumn{3}{|c|}{ Coliformes totales } & 0.0219 & 0.4971 \\
\hline \multicolumn{3}{|c|}{ Coliformes fecales } & 0.0921 & 0.0316 \\
\hline \multicolumn{5}{|c|}{ Prueba de comparación de Duncan } \\
\hline \multicolumn{5}{|c|}{ Afluentes } \\
\hline Parámetro & \multicolumn{2}{|c|}{ Coliformes fecales } & \multicolumn{2}{|c|}{ Conductividad } \\
\hline Zona & $\begin{array}{c}\text { Media } \\
\text { NMP/100 ml }\end{array}$ & $\begin{array}{c}\mathrm{E} . \\
\text { Experimental }\end{array}$ & Media uS/ $\mathbf{c m}^{2}$ & \begin{tabular}{|c|} 
E. \\
Experimental
\end{tabular} \\
\hline Alta & $386.24 \mathrm{a}$ & 225.95 & $38.67 \mathrm{~b}$ & 3.9 \\
\hline Urbana & $390.2 \mathrm{a}$ & 291.7 & $45.57 \mathrm{~b}$ & 5.03 \\
\hline Baja & $1026 \mathrm{~b}$ & 161.25 & $26.15 \mathrm{a}$ & 2.78 \\
\hline \multicolumn{5}{|c|}{ Curso principal } \\
\hline Parámetro & \multicolumn{2}{|c|}{ Conductividad } & \multicolumn{2}{|c|}{ Coliformes totales } \\
\hline Zona & Media uS $/ \mathrm{cm}^{2}$ & $\begin{array}{c}E . \\
\text { experimental }\end{array}$ & Media NMP/100 ml & $\begin{array}{c}\mathrm{E} . \\
\text { experimental }\end{array}$ \\
\hline Alta & $60.85 a b$ & 5.23 & $10313.12 \mathrm{a}$ & 10800.55 \\
\hline Urbana & $65.62 b$ & 4.53 & $44649.55 \mathrm{~b}$ & 9353.55 \\
\hline Baja & $47.97 a b$ & 5.23 & $48833.3 \mathrm{~b}$ & 10800.55 \\
\hline
\end{tabular}

a, b: valores con diferencias significativas para $p \leq 0.05$. 


\section{Tecnologíy y
Ciencias ฐAgua}

2021, Instituto Mexicano de Tecnología del Agua

Open Access bajo la licencia CCBY-NC-SA 4.0

(https://creativecommons.org/licenses/by-nc-sa/4.0/)

El análisis de correlación (Tabla 4) muestra relaciones positivas significativas al 0.01 entre la saturación de oxígeno con la demanda química y biológica de oxígeno en la zona baja, y en todas las zonas, entre coliformes totales y fecales conductividad y $\mathrm{pH}$; demanda biológica de oxígeno con $\mathrm{pH}$ y demanda química de oxígeno en todas las zonas. Se muestran correlaciones negativas significativas al 0.05 en la zona urbana entre DBO con saturación de oxígeno, coliformes totales, y en la zona baja entre coliformes totales con pH y demanda biológica de oxígeno. También se identifican relaciones significativas positivas al 0.05 entre conductividad con demanda química de oxígeno en la zona urbana y con demanda biológica de oxígeno en la zona alta. Muestran correlaciones significativas en las tres zonas al 0.01 la concentración de coliformes totales con coliformes fecales; entre $\mathrm{pH}$ y conductividad en la zona alta, y al 0.05 en las zonas urbana y baja. Muestra correlaciones significativas 0.05 entre la DQO y pH en la zona urbana; entre DBO y DQO al 0.05 en la zona alta, y al 0.01 en la zona urbana. También en referencia a la precipitación del día de muestreo con la precipitación del día anterior, muestra correlaciones significativas al 0.01 .

Tabla 4. Correlación entre parámetros.

\begin{tabular}{|c|c|c|c|c|c|c|c|c|c|c|}
\hline Zona & Parámetro & $\begin{array}{l}\text { Colif. } \\
\text { totales }\end{array}$ & $\begin{array}{l}\text { Colif. } \\
\text { fecales }\end{array}$ & Conductividad & $\mathrm{pH}$ & DQO & DBO & Sat. 02 & $\begin{array}{l}\text { Precip. D. } \\
\text { muestreo }\end{array}$ & $\begin{array}{c}\text { Precip. } \\
\text { D. } \\
\text { anterior }\end{array}$ \\
\hline \multirow{3}{*}{$\frac{\pi}{\frac{\pi}{4}}$} & Colif. totales & 1 & $.932^{* *}$ & -.135 & -.153 & -.253 & -.256 & -.149 & $.373^{*}$ & $.442^{* *}$ \\
\hline & Colif. fecales & & 1 & -.194 & -.234 & -.103 & $\begin{array}{l}.208 \\
\end{array}$ & -.106 & .314 & .329 \\
\hline & Conductividad & & & 1 & $.673^{* *}$ & .321 & .156 & .078 & -.167 & .119 \\
\hline
\end{tabular}


2021, Instituto Mexicano de Tecnología del Agua

Tecnología y

Open Access bajo la licencia CCBY-NC-SA 4.0

(https://creativecommons.org/licenses/by-nc-sa/4.0/)

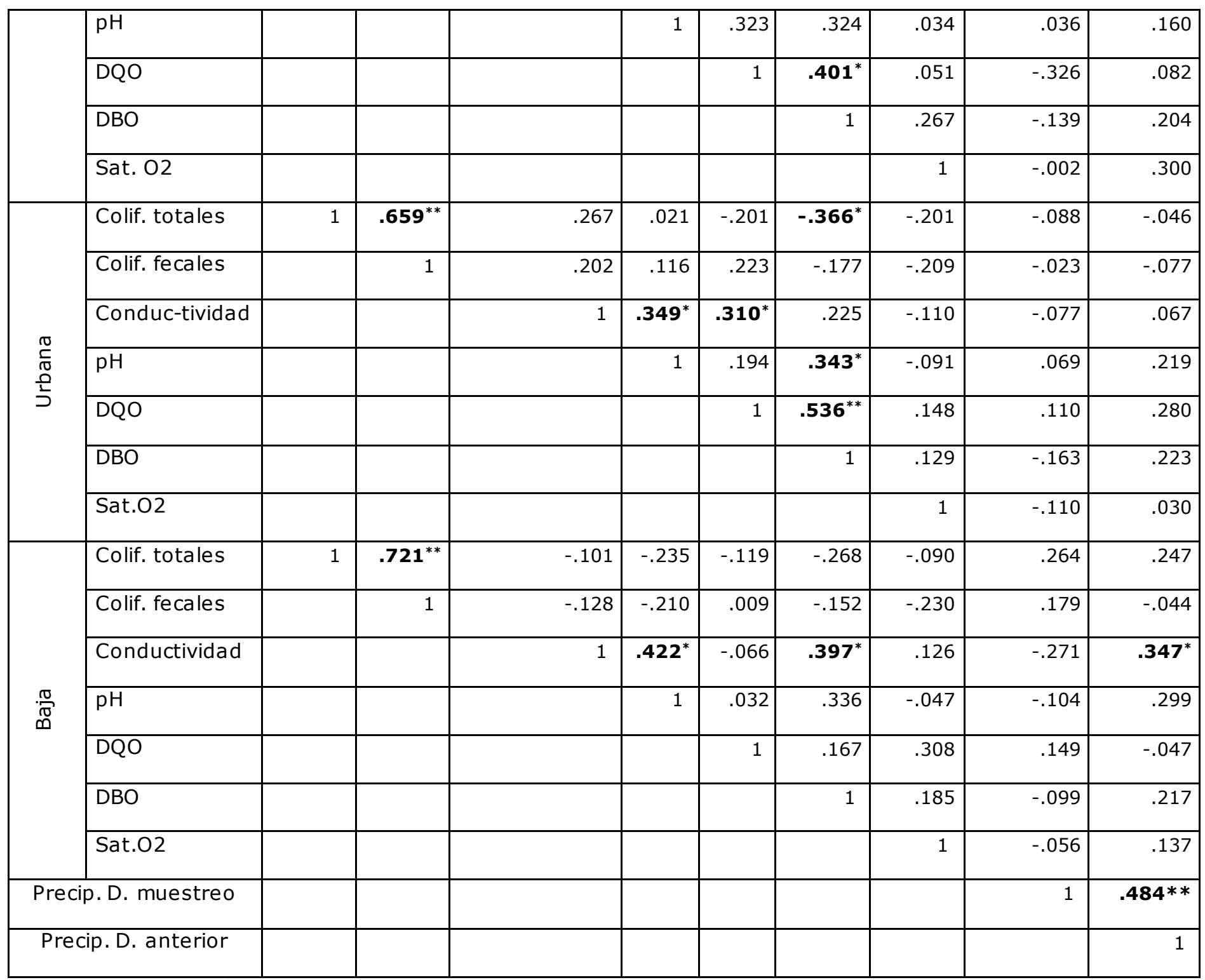

* La correlación es significativa en el nivel 0.05 (dos colas).

** La correlación es significativa en el nivel 0.01 (dos colas).

En cuanto a la precipitación del día de muestreo y del día anterior, en la zona alta muestra correlaciones significativas con coliformes totales al 0.05 el día del muestreo y 0.01 el día anterior, y al 0.05 con la conductividad y la precipitación en el día anterior en la zona baja; para este caso, las tres 


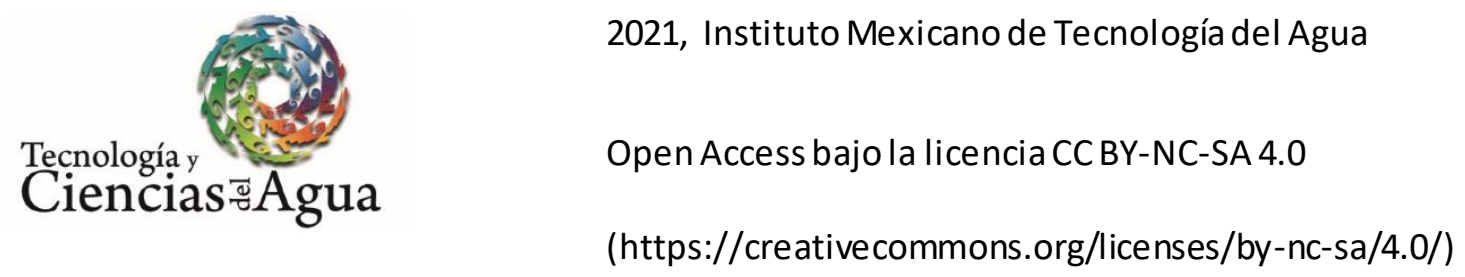

zonas reportan el mismo valor, ya que se trabajó solo con una estación meteorológica.

El resultado de las encuestas mostró que en la actividad agrícola en el curso principal (Tabla 5) predominan los cultivos de yuca, papa china y caña de azúcar; en la zona baja más, el 40 \% de los encuestados reporta este cultivo; la producción pecuaria en porcentajes inferiores muestra como principales al ganado vacuno (22\%), porcino y producción de aves $(18 \%)$, y existe predominancia de aserraderos y lubricadores, en especial en la zona urbana. En los afluentes, de la actividad agrícola destaca en la zona baja el cultivo de papa china, seguido de yuca y caña de azúcar; estos dos últimos destacan en la zona alta. En la producción pecuaria, en las zonas alta y baja destaca la producción de ganado vacuno, ganado porcino y pollos, siendo este último también importante en las zonas bajas, donde se reporta baja cantidad de pequeña industria como aserraderos y paneleras. Si bien este estudio no contemplo las áreas dedicadas a estas producciones, podemos citar a Abril, Ruiz, Alonso, Torres y Cabrera (2016), quienes mencionan en un estudio realizado en la provincia de Pastaza, que en su mayoría abarca las parroquias ubicadas en el área de estudio, la producción de caña de azúcar reporta 1403.8 ha, 141.3 ha de yuca y plátano, 284.8 ha de pastos para manejo de ganado vacuno; 17 fincas reportan tener entre 11 a 50 animales, y en mismo número de fincas de 2 a 10 cabezas de ganado vacuno; en ganado porcino, nueve fincas indican tener hasta 10 animales, y tres fincas reportan tener de 10 a 100 animales. En la producción de pollos existen producciones caseras de hasta 100 pollos, con un total de 12 fincas; de 101 a 500 pollos en una finca, y producciones de más de 2000 pollos en dos fincas.

Tabla 5. Actividades que se desarrollan en la zona y servicios sanitarios. 
2021, Instituto Mexicano de Tecnología del Agua

Tecnología y

Open Access bajo la licencia CC BY-NC-SA 4.0

(https://creativecommons.org/licenses/by-nc-sa/4.0/)

\begin{tabular}{|c|c|c|c|c|c|c|}
\hline & & $\begin{array}{l}\text { Alta } \\
\text { (\%) }\end{array}$ & $\begin{array}{c}\text { Urbana } \\
(\%)\end{array}$ & $\begin{array}{l}\text { Baja } \\
(\%)\end{array}$ & $\begin{array}{l}\text { Alta } \\
\text { (\%) }\end{array}$ & $\begin{array}{l}\text { Baja } \\
(\%)\end{array}$ \\
\hline \multirow{7}{*}{ Agrícola (cultivo) } & Caña & 4 & 8 & 40 & 20 & 39 \\
\hline & Papa china & 8 & 9 & 53 & 0 & 51 \\
\hline & Yuca & 12 & 10 & 58 & 20 & 39 \\
\hline & Plátano & 8 & 3 & 34 & 0 & 27 \\
\hline & Cacao & & 3 & 26 & & \\
\hline & Cítricos & & 2 & 11 & 0 & 3 \\
\hline & Otros & 8 & & 16 & & \\
\hline \multirow{5}{*}{ Pecuaria } & Ganado vacuno & 8 & 2 & 23 & 0 & 3 \\
\hline & Ganado porcino & 8 & 2 & 18 & 0 & 4 \\
\hline & Pollos & 4 & 1 & 18 & 20 & 7 \\
\hline & Peces & & & 4 & 20 & 3 \\
\hline & Equinos & & 1 & 12 & 0 & 1 \\
\hline \multirow{4}{*}{ Industrial } & Aserraderos & & 1 & & 0 & 1 \\
\hline & Paneleras & & & 2 & 0 & 1 \\
\hline & Lubricadoras & & 2 & 1 & & 1 \\
\hline & Otras & & 2 & 0 & & 1 \\
\hline \multicolumn{2}{|c|}{ Dispone de alcantarillado } & 8 & 25 & 6 & 20 & 18 \\
\hline \multirow{4}{*}{ Tipo de servicio } & Servicio higiénico & 28 & 42 & 9 & 80 & 18 \\
\hline & \begin{tabular}{|l} 
Letrina \\
\end{tabular} & 8 & 19 & 48 & & 33 \\
\hline & Pozo séptico & 36 & 20 & 26 & 20 & 28 \\
\hline & Otro no especificado & 20 & 10 & 13 & & 12 \\
\hline \multirow{4}{*}{$\begin{array}{l}\text { Disposición final } \\
\text { aguas servidas }\end{array}$} & Alcantarillado & 16 & 39 & 16 & 80 & 16 \\
\hline & Río & 44 & 28 & 42 & & 46 \\
\hline & Estero & 12 & 13 & 22 & 20 & 13 \\
\hline & Otro & 28 & 23 & 26 & & 3 \\
\hline
\end{tabular}




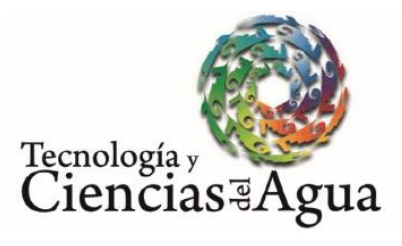

2021, Instituto Mexicano de Tecnología del Agua

Open Access bajo la licencia CCBY-NC-SA 4.0

(https://creativecommons.org/licenses/by-nc-sa/4.0/)

En el curso principal en la zona urbana, el alcantarillado llega a un 30 $\%$ de cobertura en la población; en el resto de las zonas es inferior a un 20 $\%$. La mayoría de zonas poseen servicio de recolección de residuos, pero sólo en la zona urbana predomina la recolección diaria. En el curso principal, en las zonas alta y urbana predomina el uso de servicio higiénico y pozo séptico; mientras en la zona baja se da mayor uso a la letrina; sólo en la zona urbana, el $40 \%$ de la población dispone las aguas residuales en el sistema de alcantarillado público; en las zonas alta y baja en su mayoría son dispuestas en el río o en esteros. En los afluentes, en la zona alta existe mayor uso de servicio higiénico y las aguas residuales se depositan en su mayoría al alcantarillado público; en las zonas bajas se usa mayoritariamente letrinas y pozos sépticos, y las aguas son depositadas en el río y esteros. Los resultados de los servicios básicos con los que cuentan las viviendas al ser relacionados con las concentraciones de coliformes fecales indican que en las zonas alta y baja que presentan características rurales, la deposición de materia fecal presentaría mayor cantidad de descargas con poco volumen, generando una dispersión del contaminante; mientras en la zona urbana, al ser depositado en el alcantarillado, concentra la materia fecal en el sector, recibiendo también aportes de descargas dispuestas directamente al río. Esto ratifica que la influencia del tipo de zona incide en la concentración de coliformes fecales, siendo más evidente en la zona urbana, donde se identificaron diferencias estadísticas evidenciadas en el análisis de varianza.

\section{Discusión}




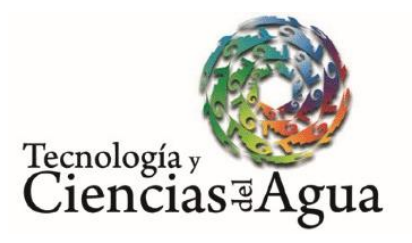

2021, Instituto Mexicano de Tecnología del Agua

Open Access bajo la licencia CCBY-NC-SA 4.0

(https://creativecommons.org/licenses/by-nc-sa/4.0/)

El estudio muestra una interacción entre el tipo de zona, donde existe una influencia de la población sobre la concentración de los diferentes parámetros; donde en la zona alta, al tener una cobertura principalmente de bosque secundario y menor cantidad de población, muestra en sus parámetros valores más ajustados a los criterios establecidos en la normativa ecuatoriana. La zona, al presentar una topografía ondulada, muestra mayor cantidad de pendientes, presenta cambios en el relieve en a partir de los $8 \mathrm{~km}$ desde la naciente en ambos ramales, donde de acuerdo con los criterios de Martínez (2006) presentan una pendiente alta (> $7 \%$ ) y media (de 2 a $7 \%$ ), lo cual influye en tener una mayor velocidad en el flujo y más presencia de saltos de agua, generando mayor oxigenación; sin embargo, el tipo de origen de cada uno de los ramales también muestra una respuesta diferente, pues el ramal Pindo nace de la acumulación de aguas de un pantano y el punto de monitoreo se encuentra a $4 \mathrm{~km}$ de la naciente; mientras que el punto cabeceras del río Puyo se encuentra en la misma naciente que proviene de fuentes subterráneas. Así, en el primer caso tiene un mayor recorrido e influye en tener una mayor saturación de oxígeno; sin embargo, en el ramal Puyo, a $8 \mathrm{~km}$ de la naciente, el punto dique de Fátima muestra valores promedios similares a los de la estación Pindo Mirador, lo cual, al considerar que aguas arriba de estos puntos no existen asentamientos poblacionales, muestra que distancia y pendiente generan estos niveles de saturación con medias del $90 \%$. Esto también se refleja en menores concentraciones de DQO, DBO5 y coliformes totales, pero no así en las concentraciones de coliformes fecales.

Al analizar en el cuerpo principal las concentraciones de coliformes totales y fecales en ambos ramales muestran un efecto de acumulación a partir de que la corriente atraviesa la zona urbana; al considerar los últimos 


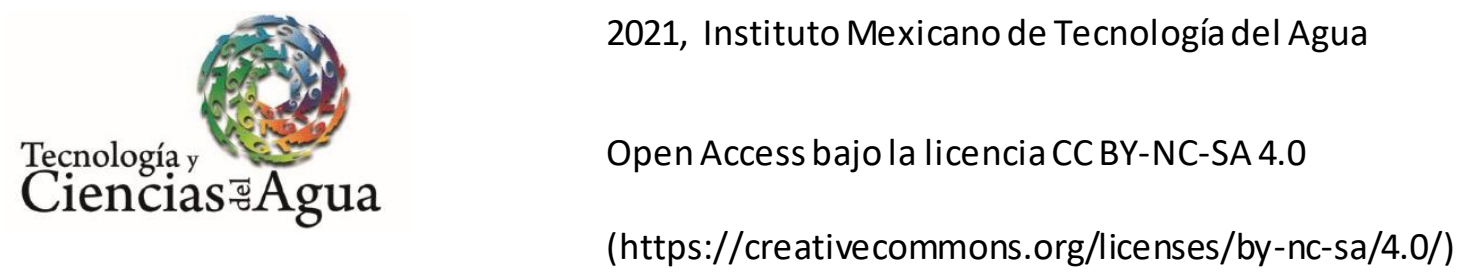

puntos en esta zona antes de la zona baja-rural, los caudales alcanzan los $7.42 \mathrm{~m} 3 / \mathrm{s}$ en el ramal Puyo y 6.03 en el ramal Pindo; esto nos plantea que tales volúmenes de agua se ven superados en su capacidad de dilución de las dos variables.

Romero, Chaile y Amoroso (2010), en estudios en ríos de montaña, presentan un incremento en el valor de potencial hidrógeno en las zonas de cuenca baja con respecto a las de cuenca alta, donde no se identifica la presencia de grandes poblaciones, lo cual no es observado en este estudio, e indica que la presencia de población influye en el comportamiento de la calidad de agua.

Pérez y Rodríguez (2008) señalan que cambios en el pH pueden indicar el ingreso de fertilizantes, de procesos de eutrofización; Marques, Albertoni y Palma-Da-Silva (2012) indican valores altos antes de la presencia de represa; en comparación con los resultados del presente estudio, en la zona alta en el curso principal reportan valores más elevados el dique de Fátima, y en los afluentes en el dique Pambay y en la zona urbana en el dique Las Palmas, los cuales tienen características de no presentar poblaciones en su curso superior.

Los valores de oxígeno disuelto son superiores al planteado por Pérez y Rodríguez (2008), quienes indican que en aguas tropicales, niveles inferiores al $55 \%$ afectan la vida acuática. Esto puede deberse a ser un río proveniente de montaña, donde la presencia de mayor cantidad de rápidos faculta el intercambio de oxígeno con el medio, aumentando su concentración.

En la demanda biológica de oxígeno, Abril, Rodríguez, Landázuri y Santi (2014), en estudios realizados entre marzo y mayo del 2012 en el dique de Shell, muestran valores entre 2 y $16 \mathrm{mg} / \mathrm{l}$ en este punto, con una 


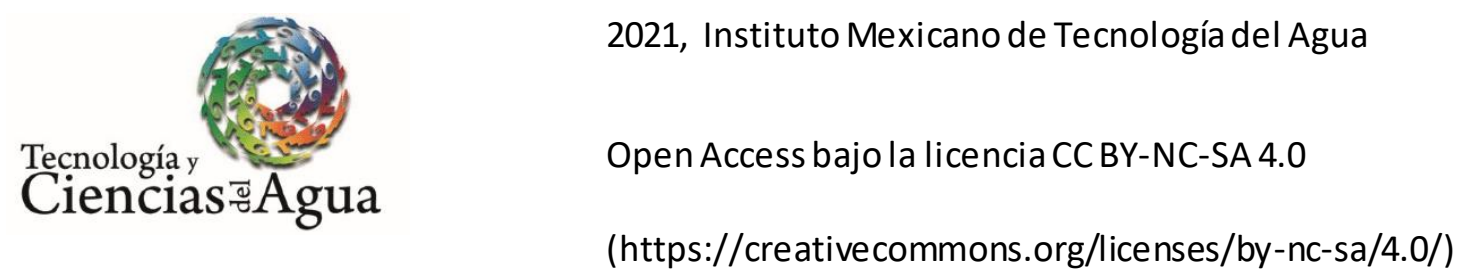

media de $9.3 \mathrm{mg} / \mathrm{l} \mathrm{ml}$, y muestra que, en las condiciones del año 2015, la presencia de sistemas de tratamiento influye en mitigar las concentraciones de dicho parámetro en este punto; en ese periodo, los valores se reportan dentro de los límites permisibles.

Abril et al. (2014), en estudios realizados entre marzo y mayo del 2012 en el dique de Shell, indican valores de coliformes totales entre 800 y $1000000 \mathrm{de}$ ufc/100 ml, con una media de 257000 ufc/100 ml, y muestran que, en condiciones del periodo evaluado, la presencia de sistemas de tratamiento influye en disminuir las altas concentraciones de este parámetro en tal punto. A partir de este punto, en lo general, el curso principal excede los límites permisibles en las zonas urbana y baja, pero en los muestreos que tuvieron mayor precipitación (marzo y abril 2015) muestra una tendencia a disminuir en las zonas bajas. Los mismos autores señalan que coliformes fecales muestran valores entre 110 y 900000 de ufc/100 ml, con una media de 228078 ufc/100 ml, e indican que, en las condiciones del año 2015, la presencia de sistemas de tratamiento influye de manera significativa en reducir las altas concentraciones de dicho parámetro en tal punto. Esto también muestra que los sistemas de tratamiento eliminan una importante carga de coliformes fecales, y su remoción es mayor en proporción a la obtenida en coliformes totales.

Marques et al. (2012) indican muestreos en zonas previas y posteriores al represamiento de agua en el río Antas en Brasil, donde la concentración de las demandas biológica y química de oxígeno disminuyen en zonas posteriores al represamiento, mientras que las concentraciones de coliformes totales y fecales se incrementan; esto se puede observar entre los puntos cercanos dique de Shell y Camal Municipal.

Torres, González Rustrián y Houbron (2013), en monitoreos realizados en el río Seco, México, con una extensión del curso de $70 \mathrm{~km}$ y 


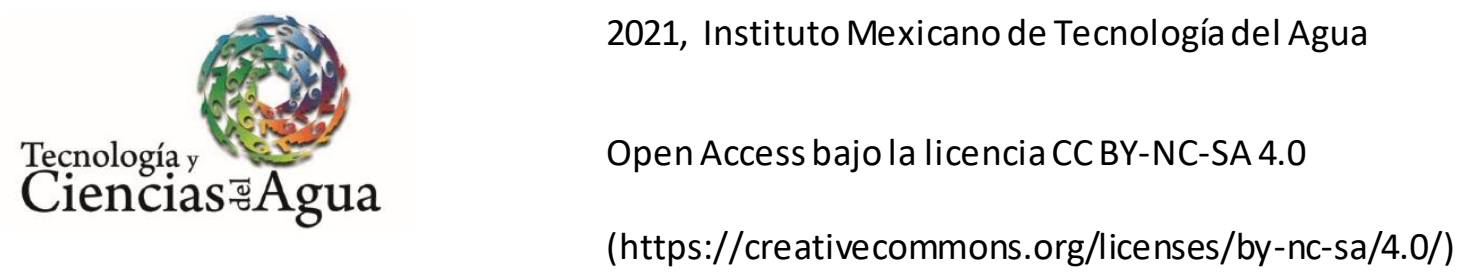

una área de cuenca de $438 \mathrm{~km}^{2}$, con precipitaciones en toda la época lluviosa de $1426 \mathrm{~mm}$ y en épocas secas de $171 \mathrm{~mm}$, que presenta características en su parte alta de pendientes del $15 \%$ hasta el primer punto de muestreo y del $3 \%$ en el resto de la cuenca, muestran las menores concentraciones de demanda química de oxígeno en el primer punto de monitoreo en la parte más alta de la cuenca; lo mismo se puede observar en el presente estudio, donde el área de cuenca es de $350 \mathrm{~km}^{2}$ y la extensión del curso de $40 \mathrm{~km}$, a pesar de presentar mayores precipitaciones.

En la zona baja, el uso de letrinas y la presencia de ganado vacuno, porcino y planteles avícolas son factores que aportan cargas de materia fecal al río. Es necesario mencionar que la presencia de estas bacterias no necesariamente representa la existencia de contaminación fecal humana, pues éstos también se encuentran en el suelo y la vegetación (Romero et al., 2010).

Se observa que las concentraciones de $\mathrm{DBO}_{5}$ (Whipple, Hunter, \& Yu, 1974) y coliformes fecales (Johnston, Detenbeck, \& Gerald, 1990) son mayores en las zonas urbanas, donde existe mayor población, lo cual se vincula con lo citado por Rörig et al. (2007).

En la correlación de variables con la precipitación del día de muestreo, sólo la concentración de coliformes totales presentó correlaciones significativas al 0.01 en la parte alta. Si aplicamos el criterio hidrológico del tiempo de concentración, el cual expresa el tiempo de recorrido de un flujo de agua entre dos puntos en una cuenca, a través de la fórmula de Kirpich (Gaspari, Rodríguez, Senisterra, Delgado, \& Besteiro, 2013), el punto dique de Fátima presenta un tiempo de 1.9 horas y el de la Estación Biológica Pindo Mirador de una hora, lo cual fundamenta que estos puntos ubicados en la parte alta presenten influencia y correlaciones significativas con 
respecto a la precipitación del día de muestreo, al recibir aportes de caudales generados por precipitaciones en horas previas.

\section{Conclusiones}

La topografía, el uso de suelo y la presencia de poblaciones influyen en las características de las concentraciones de los parámetros saturación de oxígeno, DQO, DBO5, coliformes totales y fecales en el cuerpo principal y afluentes.

En el cuerpo principal, las concentraciones de coliformes totales y fecales se incrementa en escala exponencial a partir de la zona urbana; esto se refleja en sus promedios, sobrepasando los $10000 \mathrm{NMP} / 100 \mathrm{ml}$ en ambas medidas; al tener mayores aportes de aguas residuales dispuestas en el sistema de alcantarillado y posteriormente vertidos en el curso hídrico, sobrepasa el límite establecido en la normativa. Esta tendencia no se observa en los afluentes.

Los coeficientes de variación indican que las variables concentración de coliformes totales y fecales reportan mayor variabilidad, con rangos de 100.6 a 274 en coliformes totales, y de 88.1 a 250.9 en coliformes fecales para los puntos de monitoreo; a su vez, el pH es el que menores coeficientes de variación reporta. El análisis de correlación muestra al DBO con mayor cantidad de correlaciones con el resto de variables, principalmente con la DQO, donde en la zona alta y media presenta correlaciones significativas al 


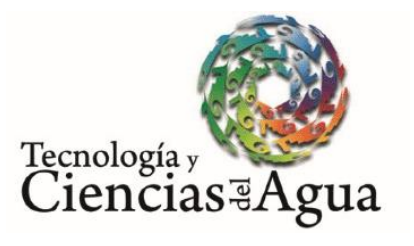

2021, Instituto Mexicano de Tecnología del Agua

Open Access bajo la licencia CCBY-NC-SA 4.0

(https://creativecommons.org/licenses/by-nc-sa/4.0/)

0.01; a su vez, la precipitación del día de muestreo significativa al 0.05 y día anterior significativa al 0.01 , presenta relación con la concentración de coliformes totales en la zona alta.

El análisis de varianza mostró diferencias para $p \leq 0.05$ en la conductividad eléctrica para el curso principal y afluentes, teniéndose menores valores en la zona baja, con una media de $47.97 \mathrm{uS} / \mathrm{cm}^{2}$ en el curso principal y $26.15 \mathrm{uS} / \mathrm{cm}^{2}$ en los afluentes; al estar esta variable relacionada con las concentraciones de sales disueltas, muestra un efecto de dilución para este parámetro. Las concentraciones de coliformes fecales en los afluentes y totales en el curso principal presentan los mayores valores en la zona baja.

La población y el tipo de disposición que dan a sus aguas residuales son el principal aporte que influye en las concentraciones de DBO, DQO, y coliformes totales y fecales, pues en las zonas urbana y baja más del $70 \%$ lo disponen al alcantarillado y río, y sólo en los sectores aledaños al dique de Shell se tienen plantas de tratamiento, lo cual también se observa en el análisis de varianza para esta variable, lo que genera un efecto acumulativo hacia la zona baja, donde se reporta mayor concentración, con una media de $48833.3 \mathrm{NMP} / 100 \mathrm{ml}$.

Las labores agrícolas y pecuarias se desarrollan principalmente en la zona baja-rural, donde en los afluentes, con base en al análisis de varianza, se reportan mayores concentraciones de coliformes fecales, con una media de $1026 \mathrm{NMP} / 100 \mathrm{ml}$.

A nivel general se recomienda que en estrategias para el manejo del recurso hídrico en zonas similares a la del estudio, los sectores ubicados en la parte alta antes de las poblaciones se deben enfocar en la preservación de los bosques; en la zona urbana, el enfoque debe direccionarse al 
tratamiento de las aguas residuales domésticas; y en la zona baja, hacia las actividades agrícolas y las aguas residuales domésticas.

\section{Agradecimientos}

Se emite un principal agradecimiento al Gobierno Autónomo Descentralizado Provincial de Pastaza y a la Universidad Estatal Amazónica por el apoyo y soporte brindado para el desarrollo de la presente investigación.

\section{Referencias}

Abril, R. V., Rodríguez, L. M., Landázuri, A. R., \& Santi, G. J. (2014). Impactos ambientales generados por el dique del río Pindo en Shell cantón Mera. Revista Amazónica de Ciencia y Tecnología, 3(3), 258283.

Abril, R. V., Ruiz, T. E., Alonso, J., Torres, V., \& Cabrera, G. (2016). Prospección de especies vegetales en la provincia de Pastaza, Ecuador. Cuban Journal of Agricultural Science, 50(4), 649-671.

AWWA Staff. (2010). Water Quality (4th ed.). Denver, USA: American Water Works Association.

Di-Rienzo, J., Casanoves, F., Balzarini, M., Gonzalez, L., Tablada, M., \& Robledo, C. (2014). INFOSTAT vers. 2014. Recuperado de http://www.infostat.com.ar

Fernández, P., \& Díaz, P. (2001). Relación entre variables cuantitativas. Unidad de Epidemiología Clínica y Bioestadística. Cuadernos de Atención Primaria, 4, 141-144. Recuperado de 
https://www.fisterra.com/mbe/investiga/var_cuantitativas /var_cuantitativas2.pdf [Citado 2016 julio]

Gaspari, F., Rodríguez, A., Senisterra, G., Delgado, M., \& Besteiro, S. (2013). Elementos metodológicos para el manejo de cuencas hidrográficas. Buenos Aires, Argentina: Editorial de la Universidad de La Plata.

GADPR Tarqui, Gobierno Autónomo Descentralizado Parroquial Rural de Tarqui. (2014). Diagnóstico de la parroquia Tarqui. Recuperado de http://app.sni.gob.ec/sni-

link/sni/PORTAL_SNI/data_sigad_plus/sigadplusdiagnostico/166001 2260001_DIAGNOSTICO TARQUI 2015_15-05-2015_18-28-31.pd

GADPR Shell, Gobierno Autónomo Descentralizado Parroquial Rural de Shell. (2015). Actualización del plan de ordenamiento y desarrollo territorial de la parroquia Shell. Recuperado de app.sni.gob.ec/snilink/sni/PORTAL_SNI/data_sigad_plus/sigadplusdiagnostico/166001 2930001_DIAGNOSTICO PD y OT SHELL 2015_15-05-2015_15-0749.pdf

GADPR Fátima, Gobierno Autónomo Descentralizado Parroquial Rural de Fátima. (2015). Plan de ordenamiento y desarrollo territorial de la parroquia Fátima. Recuperado de http://app.sni.gob.ec/snilink/sni/PORTAL_SNI/data_sigad_plus/sigadplusdocumentofinal/16 60011020001_FaPDOT_GAD\%20Parroquia2015_30-10-2015_15-22-13.pdf

GADPR Veracruz, Gobierno Autónomo Descentralizado Parroquial Rural de Veracruz. (2014). Plan de ordenamiento y desarrollo territorial de la parroquia Veracruz. Recuperado de http://app.sni.gob.ec/snilink/sni/PORTAL_SNI/data_sigad_plus/sigadplusdiagnos 
tico/1660011370001DIAGNOSTICO\%20PARROQUIA\%20VERACRUZ_ 14-05-2015_19-43-37.pdf

GADPR Pomona, Gobierno Autónomo Descentralizado Parroquial Rural de Pomona. (2015). Plan de ordenamiento y desarrollo territorial de la parroquia Pomona. Recuperado de http://app.sni.gob.ec/snilink/sni/PORTAL_SNI/data_sigad_plus/sigadplusdiagnostico/166000 8740001_PDYOT\%20POMONA\%202020_15-05-2015_23-46-25.pdf

GADPR Madre Tierra, Gobierno Autónomo Descentralizado Parroquial Rural de Madre Tierra. (2015). Plan de ordenamiento y desarrollo territorial de la parroquia Madre Tierra. Recuperado de http://app.sni.gob.ec/snilink/sni/PORTAL_SNI/data_sigad_plus/sigadplusdocumentofinal/166 0012420001_PLANF_29-10-2015_20-36-28.pdf

HACH. (2014). Oxygen Demand, Chemical (Method 8000) (9nd ed.). Recuperado de www.hach.com/asset-get.downloaden.jsa?id=7639983817

Herrera, M. (2013). Métodos estadísticos alternativos de análisis con variables discretas y categóricas en investigaciones agropecuarias (PhD. Thesis). Instituto de Ciencia Animal, Mayabeque, Universidad Agraria de la Habana.

IBM, International Business Machine. (2013). SPSS Statistic. Vers 22. Armonk, USA: International Business Machine.

Instituto Nacional de Estadísticas y Censos. (2010). Resultados del censo 2010. Recuperado de http://www.ecuadorencifras.gob.ec/wpcontent/descargas/Manu-lateral/Resultados-provinciales/pastaza.pdf 
Jadhav, H. V., \& Purohit, S. H. (eds). (2008). Global pollution and environmental monitoring. Mumbai, India: Himalaya Publishinh House.

Johnston, C. A., Detenbeck, N. E., \& Gerald, J. N. (1990). The cumulative effect of wetlands on stream water quality and quantity, a landscape approach. Biogeochemistry, 10(2), 105-141. DOI: $10.1007 /$ BF00002226

Martínez, E. (2005). Hidrología práctica (2a ed.). Madrid, España: Colegio de Ingenieros de Caminos, Canales y Puertos.

Marques, A., Marques, L., Albertoni, E., \& Palma-Da-Silva, C. (2012). Water quality in the lotic area of the Antas River before and after the construction of the Monte Claro hydroelectric plant, south Brazil. Acta Limnologica Brasiliensia, 24(3), 314-325. Recuperado de http://dx.doi.org/10.1590/S2179-975X2013005000001

Ministerio del Ambiente del Ecuador. (2015). Texto Unificado de Legislación Ambiental Secundaria (TULSMA). Fuente§ Libro VI Anexo 1. Registro Oficial Edición Especial No 316 lunes 4 de mayo de 2015.

Morales, P. (2011). Tamaño de da muestra. Recuperado de http://www.Upcomillas.Es/Personal/Peter/Investigacion/Tama\%F1o muestra.Pdf

Pérez, A. G., \& Rodríguez, A. (2008). Índice fisicoquímico de la calidad de agua para el manejo de lagunas tropicales de inundación. Revista Biología Tropical, 56(4), 1905-1918.

Rice, E., Baird, R., Eaton, A., \& Clesceri, L. (2012). Standard methods for the examination of waterand wastewater (22 ${ }^{\text {nd }}$ ed. $)$. Washington, DC, USA: American Public Health Association, American Water Works Association. 
Ritcher, B. D., Mathews, R., Harrison, D. L., \& Wigington, R. (2003). Ecologically sustainable water management. Ecological Aplications. 13, 206-224. DOI: 10.1890/10510761(2003)013[0206:ESWMMR]2.0.CO;2

Romero, N. C., Chaile, A. P., \& Amoroso, M. J. (2010). Bacteriología e hidroquímica de ríos de montaña, Tucumán. AUGMDOMUS, 2, 1626. Recuperado de http://www.revistas.unlp.edu.ar/domus/article/viewFile/128/158

Rörig, L. R., Tundisi, J. G. Schettini, C. A., Pereira-Filho, J., Menezes J. T., Almeida, T. C., Urban, S. R., Radetski, C. M., Sperb, R. C., Stramosk, C. A., Macedo, R. S., Castro-Silva, M. A., \& Perez, J. A. (2007). From a water resource to a point pollution source: The daily journey of a coastal urban stream. Brazilian Journal of Biology, 67(4), 597-609.

Torres, B., González, G., Rustrián, E., \& Houbron, E. (2013). Enfoque de cuenca para la identificación de fuentes de contaminación y evaluación de la calidad de un río, Veracruz, México. Revista Internacional de Contaminación Ambiental, 29(3), 135-146.

Whipple, W., Hunter, J., \& Yu, S. (1974). Unrecorded Pollution from Urban Runoff. Journal Water Pollution Control Federation, 46(5), 873-885. Recuperado de www.jstor.org/stable/25038730 